\title{
Ocean Dynamics and the Inner Edge of the Habitable Zone for Tidally Locked Terrestrial Planets
}

\author{
Jun Yang ${ }^{1}\left(\mathbb{D}\right.$, Dorian S. Abbot ${ }^{2}$ (i), Daniel D. B. Koll ${ }^{3}$ (1) ${\text {, Yongyun } \mathrm{Hu}^{1} \text { (ㅁ), and Adam P. Showman }}^{1,4}$ \\ ${ }^{1}$ Dept. of Atmospheric and Oceanic Sciences, School of Physics, Peking University, Beijing, 100871, People’s Republic of China; junyang@pku.edu.cn \\ ${ }^{2}$ Dept. of the Geophysical Sciences, University of Chicago, Chicago, IL 60637, USA \\ ${ }^{3}$ Dept. of Earth, Atmospheric and Planetary Sciences, MIT, Cambridge, MA 02139, USA \\ ${ }^{4}$ Dept. of Planetary Sciences and Lunar and Planetary Laboratory, University of Arizona, AZ 85721, USA \\ Received 2018 September 10; revised 2018 November 9; accepted 2018 November 14; published 2019 January 18
}

\begin{abstract}
Recent studies have shown that ocean dynamics can have a significant warming effect on the permanent night sides of 1:1 tidally locked terrestrial exoplanets with Earth-like atmospheres and oceans in the middle of the habitable zone. However, the impact of ocean dynamics on the habitable zone boundaries (inner edge and outer edge) is still unknown and represents a major gap in our understanding of this type of planet. Here, we use a coupled atmosphereocean global climate model to show that planetary heat transport from the day to nightside is dominated by the ocean at lower stellar fluxes and by the atmosphere near the inner edge of the habitable zone. This decrease in oceanic heat transport at high stellar fluxes is mainly due to weakening of surface wind stress and a decrease in surface shortwave energy deposition. We further show that ocean dynamics have almost no effect on the observational thermal phase curves of planets near the inner edge of the habitable zone. For planets in the habitable zone middle range, ocean dynamics move the hottest spot on the surface eastward from the substellar point. These results suggest that future studies of the inner edge may devote computational resources to atmosphere-only processes such as clouds and radiation. For studies of the middle range and outer edge of the habitable zone, however, fully coupled oceanatmosphere modeling will be necessary. Note that due to computational resource limitations, only one rotation period (60 Earth days) has been systematically examined in this study; future work with varying rotation periods, as well as other parameters such as atmospheric mass and composition, is required.
\end{abstract}

Key words: astrobiology - methods: numerical - planetary systems - planets and satellites: general - radiative transfer

\section{Introduction}

The ocean has a profound effect on the variation and time mean features of the climate of Earth through modifying the surface heat capacity, transporting heat from low latitudes to high latitudes, and storing carbon (Vallis 2012; Watson et al. 2015). The tight interaction between ocean, atmosphere, ice, and clouds further influences global and regional energy balances and surface temperatures. For instance, if Earth's global ocean circulation were artificially turned off, the global-mean surface temperature would decrease by several degrees (Winton 2003; Herweijer et al. 2005). How ocean dynamics influence the climate and habitability of exoplanets remains relatively unstudied.

For the past 20 years, various three-dimensional (3D) atmosphere-only climate models have been employed to examine the important effects of atmospheric circulation on the climate and habitability of 1:1 tidally locked ("synchronously rotating") terrestrial planets (e.g., Joshi et al. 1997; Merlis \& Schneider 2010; Edson et al. 2011; Pierrehumbert 2011; Leconte et al. 2013a, 2013b; Yang et al. 2013, 2014; Wang et al. 2014, 2016; Way et al. 2015; Kopparapu et al. 2016, 2017; Popp et al. 2016; Turbet et al. 2016; Boutle et al. 2017; Haqq-Misra et al. 2017; Salameh et al. 2017; Wolf 2017; Wolf et al. 2017; Bin et al. 2018; Turbet et al. 2018). These studies employed a dry land surface with no ocean or an immobile thermodynamic ocean with no ocean dynamics. The effect of ocean dynamics on exoplanets has only been addressed by a few studies (Yang et al. 2013, 2014; Hu \& Yang 2014; Cullum et al. 2014, 2016; Way et al. 2015, 2018; Del Genio et al. 2019). Studies on synchronously rotating planets have found that ocean dynamics could significantly warm the permanent night sides of planets in the middle range of the habitable zone (Hu \& Yang 2014 and Del Genio et al. 2019, or see Section 3.1 for more detailed feedback analyses). A critical unaddressed question is: could ocean dynamics have a significant effect on the location of the inner edge of the habitable zone? If ocean dynamics have a strong warming effect on planets near the inner edge, as they do for those in the middle range, this could shrink the habitable zone. As a result, the number of potentially habitable planets would be lower, making it harder to detect and study such planets in the future. Here we try to answer this question through a series of 3D climate experiments.

The maximum stellar flux investigated by $\mathrm{Hu} \&$ Yang (2014), $1400 \mathrm{~W} \mathrm{~m}^{-2}$, is only about $40 \%-50 \%$ of the stellar flux at the inner edge, according to results from atmosphere-only models (Yang et al. 2014; Kopparapu et al. 2017; Bin et al. 2018). Our new experiments show that the day-to-night oceanic heat transport (OHT) at the higher stellar fluxes of planets near the inner edge is much weaker than that for a stellar flux of $1400 \mathrm{~W} \mathrm{~m}^{-2}$ (see Section 3.2) and that the location of the inner edge would not be shifted by ocean dynamics, at least for a rotation period of 60 Earth days. Section 2 addresses the model description and experimental design. We show the results in Section 3, including the effect of ocean dynamics on planets in the middle range (Section 3.1) and near the inner edge (Section 3.2) of the habitable zone, the effect of ocean dynamics on observational thermal phase curves (Section 3.3), and an exception to our result that OHT tends to monotonically decrease with increasing stellar flux (Section 3.4). We further 
Table 1

Summary of the Climate Simulations Performed Using CCSM3

\begin{tabular}{|c|c|c|}
\hline Group & Runs & Experimental Design \\
\hline Control & 1 & $\begin{array}{l}\text { The surface is covered by an ocean with a uniform depth of about } 4000 \mathrm{~m} \text {. The stellar flux is } 1400 \mathrm{~W} \mathrm{~m}^{-2} \text { with a } 4500 \mathrm{~K} \text { blackbody } \\
\text { spectrum. Rotation period (=orbital period) is } 60 \text { Earth days. }\end{array}$ \\
\hline Stellar flux & 9 & Same as "Control" except the stellar flux is varied between 1400 and $2800 \mathrm{~W} \mathrm{~m}^{-2}$ in increments of $200 \mathrm{~W} \mathrm{~m}^{-2}$. \\
\hline Initial state & 2 & $\begin{array}{l}\text { Same as "Control" except stellar flux is set to } 1800 \mathrm{~W} \mathrm{~m}^{-2} \text { and the initial state is from the equilibrium state of the case of } \\
2400 \mathrm{~W} \mathrm{~m}^{-2} \text {, or the stellar flux is set to } 2400 \mathrm{~W} \mathrm{~m}^{-2} \text { and the initial state is from the equilibrium state of the case of } 1800 \mathrm{~W} \mathrm{~m}^{-2} \text {. }\end{array}$ \\
\hline Ocean depth & 3 & $\begin{array}{l}\text { The ocean depth is set to } 4000,800 \text {, and } 400 \mathrm{~m} \text {, respectively. The stellar flux is } 1400 \mathrm{~W} \mathrm{~m}^{-2} \text { with a } 3400 \mathrm{~K} \text { blackbody spectrum. } \\
\text { Rotation period (=orbital period) is } 37 \text { Earth days. }\end{array}$ \\
\hline Land-sea distribution & 2 & $\begin{array}{l}\text { One-ridge world: a thin barrier that completely obstructs ocean flows running from pole to pole on the eastern terminator. Two-ridge } \\
\text { world: two thin barriers on the western and eastern terminators. Other parameters are the same as the "ocean depth" cases. }\end{array}$ \\
\hline
\end{tabular}

discuss the results and suggest future required studies in Section 4, and summarize in Section 5.

\section{Model Description and Experimental Design}

We use the Community Climate System Model CCSM3, which has four coupled components: atmosphere, ocean, land, and sea ice (Collins et al. 2006). The model was developed to investigate the climates of present, past, and future Earth. We have modified the model to be able to simulate the climates of Earthlike exoplanets that have different stellar spectra, planetary orbits, masses, and land-sea distributions (Liu et al. 2013; Hu \& Yang 2014; Yang et al. 2014). By default, we use a planetary surface nearly completely covered with an ocean of water (an aqua-planet) with a uniform depth of $\simeq 4000 \mathrm{~m}$, close to the mean depth of Earth's oceans. We include two small islands at the south and north poles (poleward of $85^{\circ} \mathrm{S}(\mathrm{N})$ ) because the poles of the ocean grid have to reside on continents (Rosenbloom et al. 2011). These small-area islands should have a negligible influence on the results. ${ }^{5}$ Aqua-planet simulations provide a standard framework for understanding large-scale ocean circulations and for relating our results to previous work, including analytical theories (Smith et al. 2006; Marshall et al. 2007). Also, due to the lack of complex land barriers, an aqua-planet represents the simplest fully $3 \mathrm{D}$ and coupled system in which to investigate ocean circulation and ocean-atmosphere interaction, and therefore is most appropriate for examining the mechanisms that strengthen or weaken the ocean's effect at different stellar fluxes.

The atmosphere is assumed to be Earth-like, composed of $\mathrm{N}_{2}$ and $\mathrm{H}_{2} \mathrm{O}$, with a surface pressure of approximately $1.0 \mathrm{bar}$ (depending on the water vapor concentration). The concentrations of $\mathrm{CO}_{2}, \mathrm{CH}_{4}, \mathrm{~N}_{2} \mathrm{O}, \mathrm{O}_{2}, \mathrm{O}_{3}$, and CFCs are set to zero. Water vapor is the only greenhouse gas. We chose one sample star with a surface temperature of $4500 \mathrm{~K}$. The rotation period of the planet is 60 Earth days (=orbital period), and the stellar fluxes we tested are $1400,1600,1800,2000,2200,2400,2600$, and $2800 \mathrm{~W} \mathrm{~m}^{-2}$. The maximum stellar flux for which the model can achieve a quasi-equilibrium state is $2800 \mathrm{~W} \mathrm{~m}^{-2}$. The radius and gravity of the simulated planet are set to typical values of a super-Earth (such as the unconfirmed exoplanet Gl 581g (Vogt et al. 2010)

Del Genio et al. (2019) used another coupled atmosphere-ocean circulation model, ROCKE3D, to examine the possible climate scenarios of a tidally locked planet-Proxima Centauri b-and employed an aqua-planet configuration without any continent in most of their simulations. The main characteristics of ocean circulation (ocean currents, spatial patterns of sea surface temperature and sea ice, etc.) in their simulations are similar to our results. and the confirmed exoplanet LHS 1140b (Dittmann et al. 2017)): 1.5 times Earth's radius and 1.38 times Earth's gravity. Both obliquity and eccentricity are set to zero.

In our simulations, for a given stellar temperature, the rotation period is fixed when varying the stellar flux, as was done by Wang et al. (2014), Way et al. (2015), and Fujii et al. (2017). The period of 60 Earth days we chose is close to the rotation period near the inner edge of the habitable zone for a star with a temperature of $4500 \mathrm{~K}$ (see Figure 2 of Kopparapu et al. 2016). This design allows us to isolate the effect of increasing stellar radiation and is sufficient to demonstrate the role of ocean dynamics on the inner edge of the habitable zone. Our setup is different from that employed by Kopparapu et al. (2016), Haqq-Misra et al. (2017), and Wolf et al. (2017), who modified the rotation period and stellar flux simultaneously. Their experiments are able to self-consistently consider the combined effect of the Coriolis force and stellar flux, but do not allow separate consideration of each factor. In the future, it would be interesting to investigate the combined effect of varying the rotation period and stellar flux using a coupled atmosphere-ocean model.

In order to test the effect of continent and ocean depth on the ocean circulation, we also carried out experiments with a one-ridge or a two-ridge continental setup ${ }^{6}$ or different ocean depths, 800 and $400 \mathrm{~m}$ (Table 1). The geothermal heat flux is set to zero, and the only energy source is stellar radiation. We find our results are robust across these parameters but future work is required to determine whether our results are strongly sensitive to additional effects, such as other resonant orbitalrotational states, different planetary radii and gravities, different land-sea distributions, and other ocean depths.

In the ocean component of our model, diffusion and viscosity parameters are assumed to be the same as for Earth's present-day oceans. The parameterization of along-isopycnal potential temperature and salinity diffusion uses the Gent-McWilliams scheme (Gent \& McWilliams 1990) with a coefficient of $800 \mathrm{~m}^{2} \mathrm{~s}^{-1}$. Horizontal viscosity in the momentum equation employs the anisotropic formulation (Smith \& McWilliams 2003). Diapycnal

\footnotetext{
6 The ocean depth and the land-sea distribution experiments were run about 3 years ago, while the other experiments were run this year and employed somewhat different stellar temperatures and rotation periods (Table 1). However, the differences in stellar temperature and rotation period should have a very small effect on the results here and do not influence the conclusion of this paper. This is due to that fact that (1) both 37 days and 60 days are in the slowly rotating regime (Edson et al. 2011; Haqq-Misra et al. 2017) and (2) although the stellar spectrum can influence the surface albedo of ice and snow, the dayside is ice-free in most of the CCSM3's experiments.
} 
mixing is represented by the K-profile parameterization boundarylayer scheme (Large et al. 1990). Several physical processes are considered in the scheme, including internal waves, shear instability, convective instability and double diffusion (Smith \& Gent 2004). Separate studies using high-resolution eddy-resolving ocean models are required to understand the uncertainties of these parameters for Earth-like exoplanets; computational resource limitations preclude such sensitivity experiments at present.

The atmosphere and land components of the model have a horizontal resolution of $3.75^{\circ} \times 3.75^{\circ}$, with 26 vertical levels from the surface to $\sim 36 \mathrm{~km}$. The ocean and sea-ice components have a variable latitudinal resolution starting at $0.9^{\circ}$ near the equator, a constant longitudinal resolution of $3.6^{\circ}$, and 25 vertical levels. By default, the time step for the atmosphere and land components is set to $900 \mathrm{~s}$. For the simulations with high stellar fluxes, we used a smaller time step to avoid numerical instability; the minimum time step we examined is $60 \mathrm{~s}$. Due to computational limitations, time steps less than $60 \mathrm{~s}$ were not tested. For the ocean, the time step is two hours. The coupling time interval between the atmosphere and ocean is one Earth day. The sea ice albedo is 0.50 in the visible and 0.30 in the near-infrared. The snow albedo is 0.91 in the visible and 0.63 in the near-infrared (Briegleb et al. 2002). The broadband surface albedo, therefore, depends on stellar spectrum and is less reflective at redder wavelengths (Joshi \& Haberle 2012; Shields et al. 2013).

The atmosphere was initialized from a state close to modern Earth, and the ocean was initialized from a state of rest with a horizontally uniform temperature. We integrated each case for about 1000 or 2000 Earth yr, and present the final 100 or $200 \mathrm{yr}$ in the following analyses. Time-series of global-mean surface temperature, energy balance (absorbed shortwave radiation minus outgoing longwave radiation) at the top of the atmosphere, vertically averaged ocean potential temperature, and sea ice coverage are shown in Figures 1(a)-(d). In most cases, the system reaches quasi-equilibrium within about $1000 \mathrm{yr}$, although several simulations exhibit significant oscillations after that time: (1) the 2000 and $2600 \mathrm{~W} \mathrm{~m}^{-2}$ cases show strong oscillations with a period of $\simeq 100$ Earth yr; (2) the 1400 and $1600 \mathrm{~W} \mathrm{~m}^{-2}$ cases also exhibit significant variations but with a period of $\simeq 30$ Earth yr; (3) the $2400 \mathrm{~W} \mathrm{~m}^{-2}$ case shows irregular rises in sea ice coverage and falls in sea temperature and ocean potential temperature. We speculate that these long-time oscillations may arise from the interactions between atmosphere, sea ice, and ocean (especially Rossby and Kelvin waves), such as that shown in Marshall et al. (2007); detailed analyses of the underlying mechanisms are beyond the scope of the present work. In this paper, we will focus on the mean state only. Note that ocean potential temperature in the $2800 \mathrm{~W} \mathrm{~m}^{-2}$ case is still increasing although the surface temperature does not show a significant warming trend; the model blew up when we tried to further integrate the run. If the model could be integrated longer, the ocean would become warmer, especially in relatively cold regions at the ocean bottom and the ocean in the nightside, so that OHT from the dayside to the nightside (see Section 3.2 below) would likely be even smaller.

In the following section, we will analyze our equilibrated CCSM3 simulations to understand the ocean's effect on the planetary climates. To isolate the effect of ocean dynamics, we preformed corresponding atmosphere-only experiments using the Community Atmosphere Model version 3.1 (CAM3, Collins et al. 2004), which is the atmosphere component of CCSM3. CAM3 is coupled with a $50 \mathrm{~m}$ mixed layer, immobile ocean, and ocean heat transport is specified to be zero everywhere. The model is coupled to a thermodynamic sea ice model, in which sea ice flows are not considered. Each case is integrated for about 60 Earth years and reaches a steady state after about $40 \mathrm{yr}$. Averages over the last $5 \mathrm{yr}$ are used for our analyses. The maximum stellar flux for which simulations in CAM3 can achieve a quasi-equilibrium state is $3000 \mathrm{~W} \mathrm{~m}^{-2}$, higher than that in CCSM3.

\section{Results \\ 3.1. The Ocean's Strong Effect in the Middle of the Habitable Zone}

The ocean transports heat from the substellar region where there is net energy gain to the nightside where there is net energy loss. As a result, the nightside in CCSM3 is much warmer than that in CAM3 by 40-50 K (Figure 2; see also Hu \& Yang 2014 and Del Genio et al. 2019). The ocean also transports heat in the north-south direction and thereby warms the dayside high latitudes. Despite the ocean transporting heat away from the substellar region, surface temperatures there decrease only slightly, by $\simeq 10 \mathrm{~K}$. This is mainly due to a negative cloud feedback: as the OHT carries energy away from the substellar region, the surface temperature decreases and therefore convection over the substellar region becomes weaker, so that cloud optical depth (Figure 2(e)) and planetary albedo decrease, allowing more stellar energy to reach the surface and warm it. This feedback is similar to that described in Koll \& Abbot (2013). Moreover, per unit of energy, the warming of the nightside surface should be greater than the cooling of the dayside surface because of the nonlinear dependence of thermal emission on temperature (i.e., the Planck effect) and the fact that the nightside surface is colder than the dayside surface.

Besides the direct effects of ocean dynamics, feedbacks associated with sea ice, water vapor, and atmospheric lapse rate further act to warm the surface. OHT melts the sea ice around the terminators and at the dayside high latitudes (Figure 2(c)), reducing the planetary albedo. Meanwhile, in the experiments with stellar fluxes equal to or less than $1600 \mathrm{~W} \mathrm{~m}^{-2}$, sea ice in CCSM3 is only several meters thick, being 2-3 orders of magnitude thinner than the nightside sea ice thickness in CAM3, similar to the results in Yang et al. (2014), so OHT is effective at preventing water trapping on the nightside. For stellar fluxes higher than $1600 \mathrm{~W} \mathrm{~m}^{-2}$, there is no snow or ice either on the day or nightside in CCSM3, although in CAM3 the nightside is still covered by ice and snow. Compared to CAM3, CCSM3's water vapor concentration is higher on the nightside (Figure 2(d), although lower in the substellar region), the temperature inversion (i.e., air temperature being higher than the surface, Leconte et al. 2013b; Zalucha et al. 2013; Yang \& Abbot 2014) disappears on the nightside (Figure 2(f)), and the longwave cloud radiative effect is higher (a warming effect, Figure 3). All of these factors act to increase the atmospheric greenhouse effect on the nightside in CCSM3. In contrast, on the nightside in CAM3, clouds form at the layers near the temperature inversion (Figure 2(f)), which acts to trap water vapor that evaporated from the surface; this mechanism is similar to the low-level stratus cloud formation over the eastern subtropical Pacific Ocean where the sea surface is cooler than the average sea surface due to ocean upwelling there (chapter 3.13 of Hartmann 2016). These clouds emit longwave radiation to space at temperatures higher than the surface, causing a negative longwave cloud radiative effect on the nightside (Figure 3(b)) and inducing a cooling effect on the air and the 

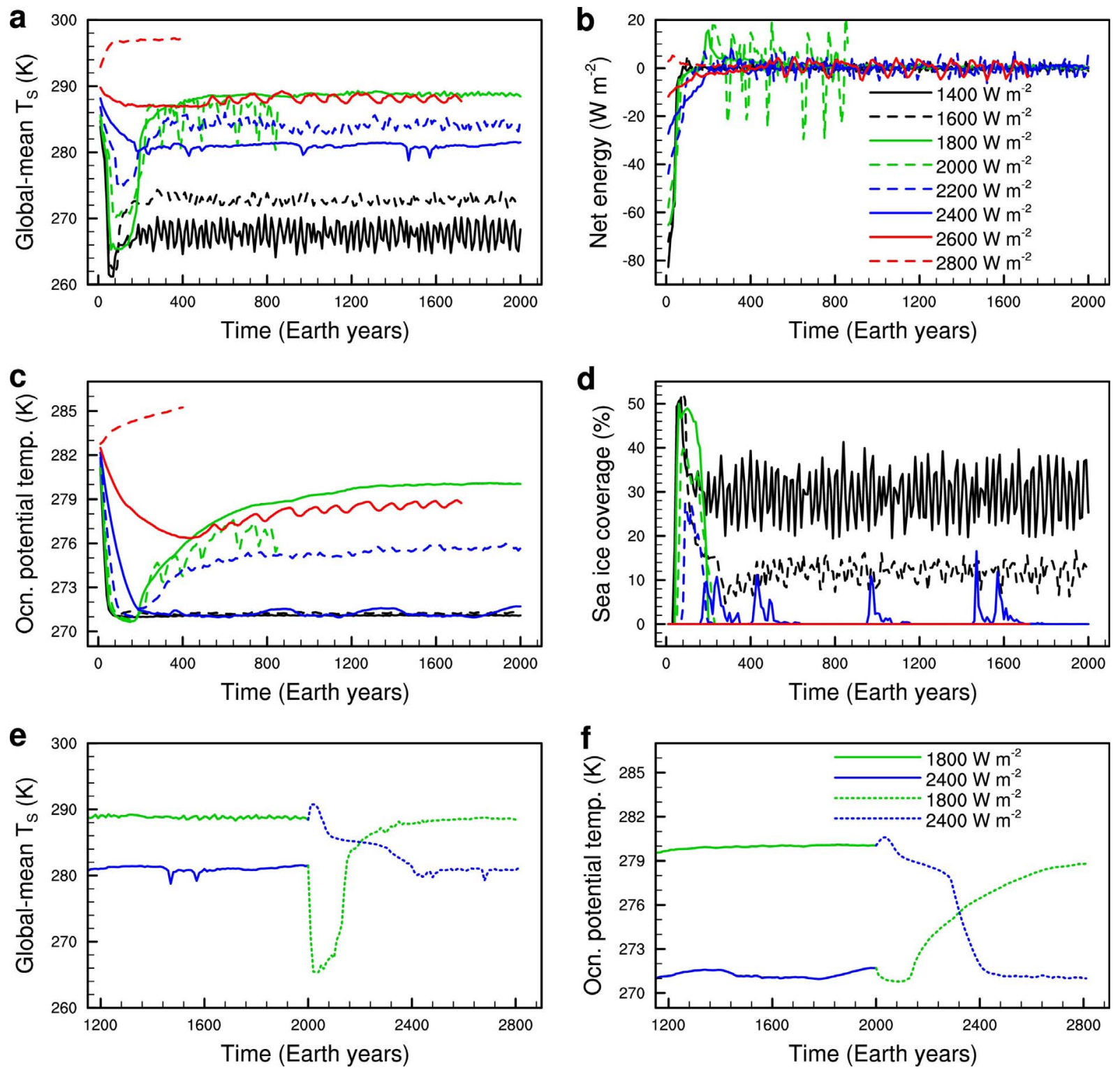

Figure 1. Time-series of global-mean characteristics of the CCSM3 simulations: (a) surface air temperature; (b) net energy flux (shortwave minus longwave) at the top of the atmosphere; (c) vertically averaged ocean potential temperature; and (d) sea ice coverage. The stellar flux is increased from 1400 to $2800 \mathrm{~W} \mathrm{~m}^{-2}$ in increments of $200 \mathrm{~W} \mathrm{~m}^{-2}$. (e)-(f) Independence of the equilibrium state from the initial state, for the stellar fluxes of 1800 and $2400 \mathrm{~W} \mathrm{~m}^{-2}$; (e) global-mean surface temperature; and (f) global- and vertical-mean ocean potential temperature. In (e)-(f), the solid lines are the same as those shown in (a) and (c), and the dotted lines are initialized from the equilibrium states of the solid lines. Each data point in these time-series is the average over 10 Earth yr. In all of these experiments, the rotation period is 60 Earth days and we apply a $4500 \mathrm{~K}$ blackbody spectrum and an aqua-planet with a uniform depth of $\simeq 4000 \mathrm{~m}$.

surface, except for the case of $3000 \mathrm{~W} \mathrm{~m}^{-2}$, in which the temperature inversion disappears. In CCSM3, there is no temperature inversion in all of the experiments shown here (note that for a much lower stellar flux, such as $625 \mathrm{~W} \mathrm{~m}^{-2}$ in which ocean heat transport is very weak, there is a temperature inversion (figure not shown)) and the longwave cloud radiative effect is positive (warming) everywhere.

The direct effect of ocean dynamics is therefore to reduce the day-to-night surface temperature contrast in CCSM3 compared to CAM3. Associated surface and atmospheric feedbacks then amplify the nightside warming, which is why global-mean surface temperature in CCSM3 is much higher than that in CAM3 (Figure 2(b)). However, as we show in the next section, these differences become smaller and smaller as the stellar flux is increased.

\subsection{Decreasing Trend of OHT with Increasing Stellar Flux}

As the stellar flux is increased, the global-mean surface temperature (as well as the maximum and minimum surface temperatures) in CCSM3 generally increases, but the areaaveraged day-to-night OHT decreases when the stellar flux is higher than $1800 \mathrm{~W} \mathrm{~m}^{-2}$ (Figure 4, for a discussion of the nonmonotonic behavior of CCSM3's results, see Section 3.4). This finding seems counterintuitive because as the energy received by the dayside of the planet increases, one might expect the ocean to transport more heat to the nightside. This intuition does hold for the atmospheric and total (oceanic plus atmospheric) heat transports, which do increase with stellar flux (Figure 4(b)), but not for the OHT.

Figure 4(b) indicates that there is a compensation between OHT and atmospheric heat transport, but the compensation is 


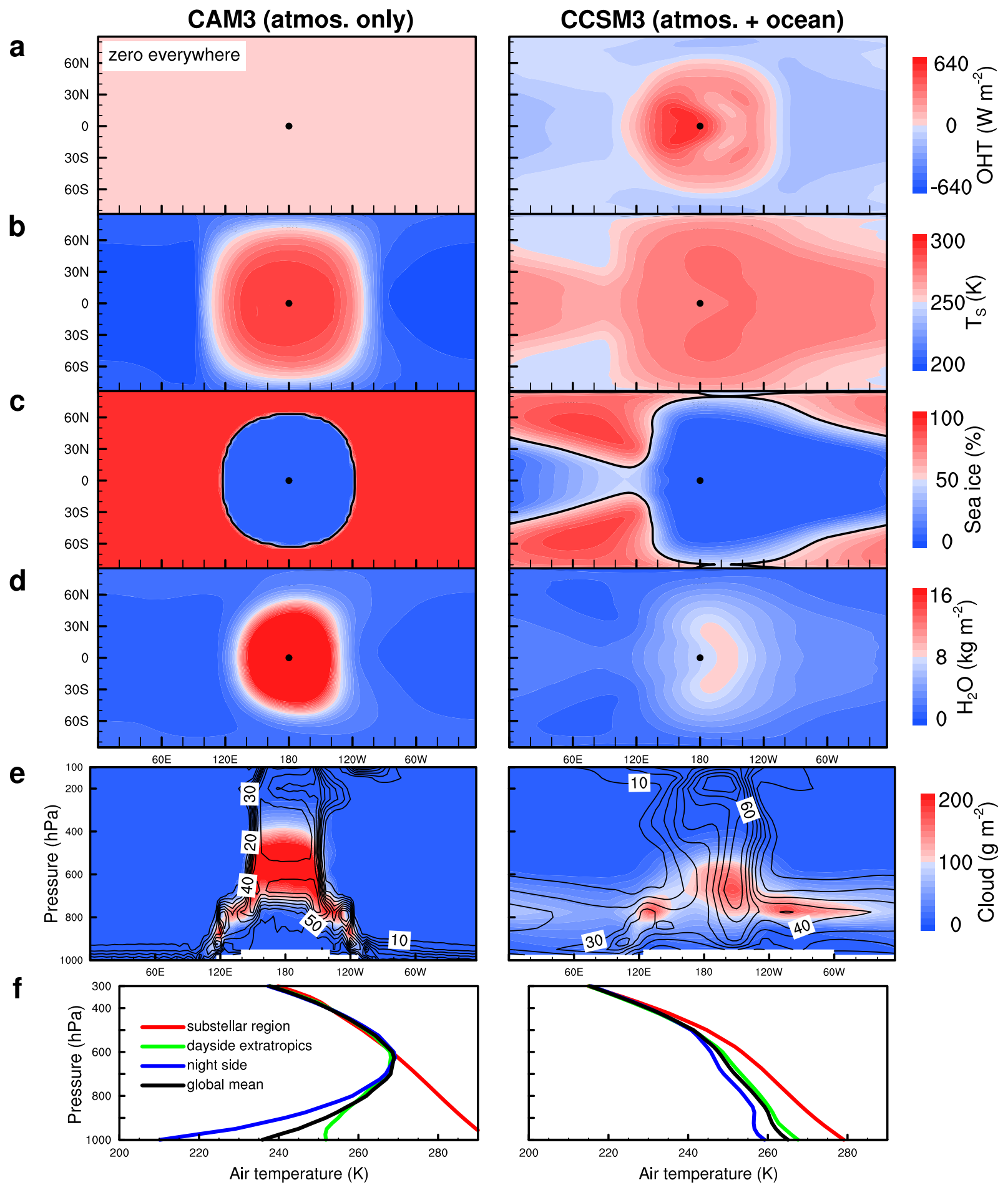

Figure 2. Strong effects of ocean dynamics on a tidally locked aqua-planet in the middle range of the habitable zone. Left: CAM3; right: CCSM3. (a) OHT (i.e., surface net heat flux; negative: heat from ocean to atmosphere; positive: heat from atmosphere to ocean); (b) surface temperature; (c) sea ice coverage (the black line shows 50\% coverage); (d) vertically integrated water vapor amount; (e) meridional-mean cloud water amount (color shading) and cloud fraction (\%, contour lines with an interval of 10\%); and (f) vertical temperature profiles for different regions. Note the vertical axis is latitude in (a)-(d) but air pressure in (e)-(f). In both experiments, the stellar flux is $1400 \mathrm{~W} \mathrm{~m}^{-2}$. In this figure and elsewhere, the substellar point is at $\left(180^{\circ}, 0^{\circ}\right)$ and is marked with a black dot.

imperfect. In CAM3, the OHT is zero and the atmospheric heat transport is higher than that in CCSM3. The total heat transport, however, is higher in CCSM3 than that in CAM3. The main reason is that the planetary albedo in CCSM3 is smaller (Figure 4(i)), meaning that more stellar radiation is absorbed by the dayside atmosphere and surface; therefore more energy can be supplied to transport by the atmosphere and ocean. This result is consistent with previous results (Enderton \& Vallis 2009; Vallis \& Farneti 2009; Farneti \& Vallis 2013): increased atmospheric heat transport generally picks up the slack of reduced OHT, but the compensation is often not $100 \%$.

The decreasing trend of OHT with increasing stellar flux results from weaker ocean currents combined with less stellar radiation 

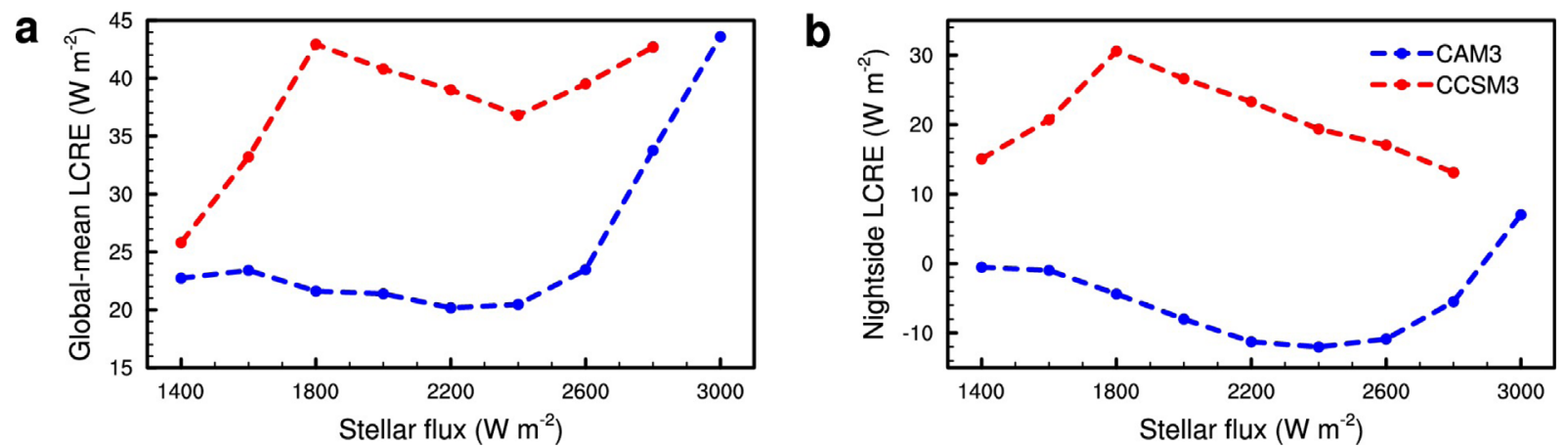

Figure 3. Longwave cloud radiative effect (LCRE) at the top of the atmosphere. Blue: CAM3; red: CCSM3. (a) Global mean; (b) nightside average.

a

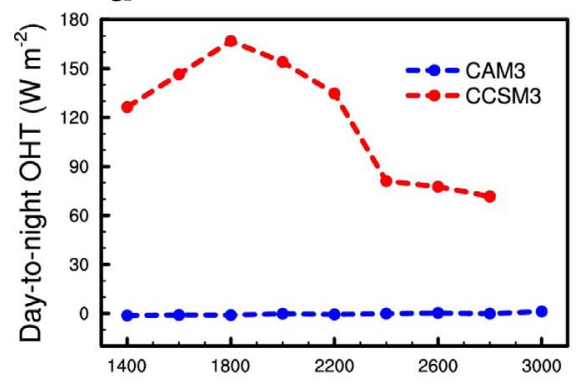

d
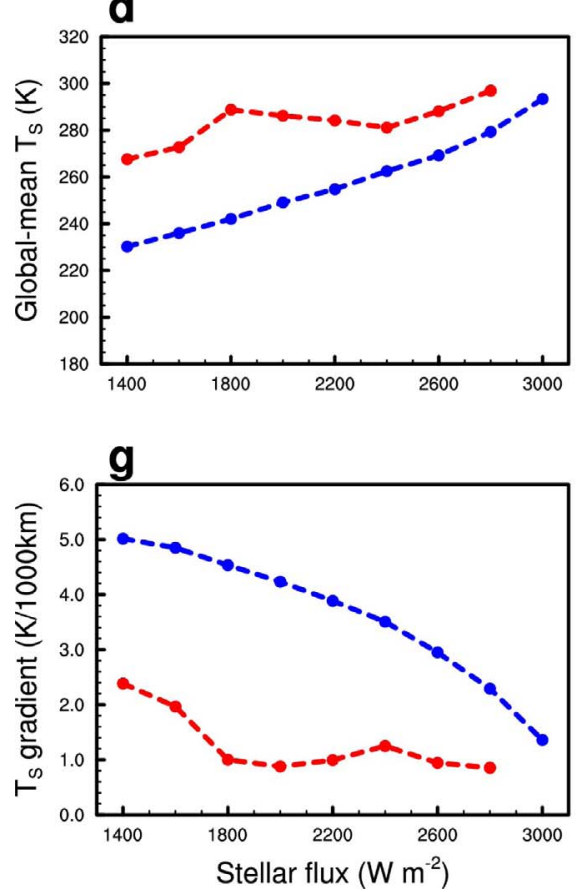

b
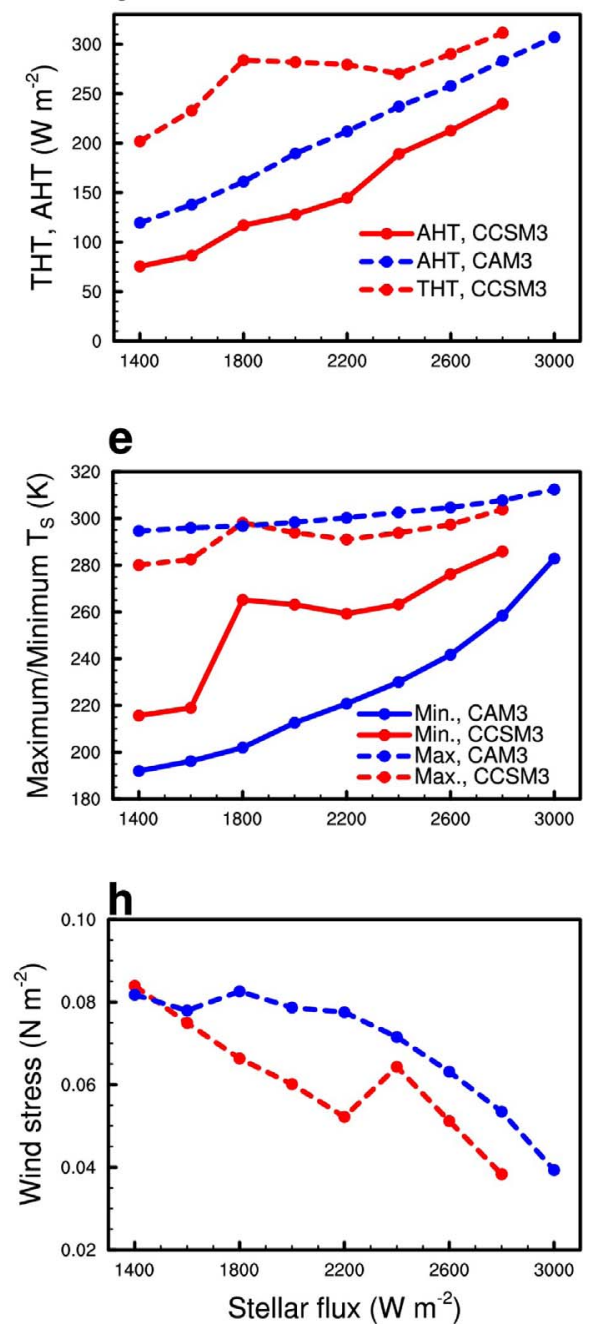

C
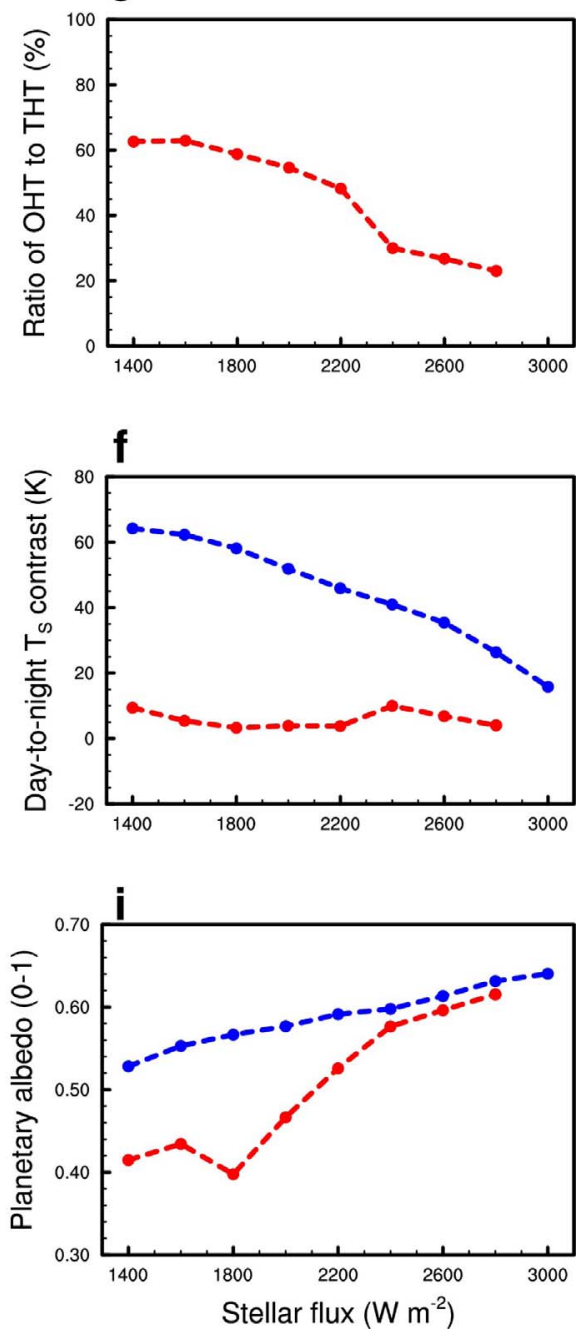

Figure 4. The effect of ocean dynamics as a function of stellar flux. Blue line: the atmosphere-only model CAM3; red line: the coupled ocean-atmosphere model CCSM3. (a) Area-averaged day-to-night oceanic heat transport (OHT); (b) day-to-night atmospheric heat transport (AHT) and total heat transport (THT); (c) the ratio of OHT to THT in CCSM3; (d) global-mean surface temperature $\left(T_{S}\right)$; (e) maximum surface temperature (dashed line) and minimum surface temperature (solid line); (f) day-to-night area-averaged $T_{S}$ contrast; (g) strength of surface temperature gradient $\left(\sqrt{\left(\frac{\partial T_{S}}{\partial x}\right)^{2}+\left(\frac{\partial T_{S}}{\partial y}\right)^{2}}\right.$ in units of $\mathrm{K}$ per $\left.1000 \mathrm{~km}\right)$; (h) strength of surface wind stress ( $\sqrt{\tau_{x}^{2}+\tau_{y}^{2}}$, where $\tau_{x}$ is the zonal wind stress and $\tau_{y}$ is the meridional wind stress); and (i) planetary albedo, as a function of stellar flux. In all of the experiments, the rotation period is 60 Earth days and the stellar spectrum is a $4500 \mathrm{~K}$ blackbody.

depositing energy at the dayside sea surface. The main characteristic of ocean circulation on a tidally locked aqua-planet is west-east currents along the equator (Figures $5(\mathrm{i})-(\mathrm{p}), \mathrm{Hu} \&$ Yang 2014). The ocean currents in the tropics result from the combined effect of surface wind stresses and equatorward momentum transport by large-scale Rossby and Kelvin waves in the ocean (more comprehensive analyses and detailed dynamical diagnostics will be addressed in a separate paper by 
a

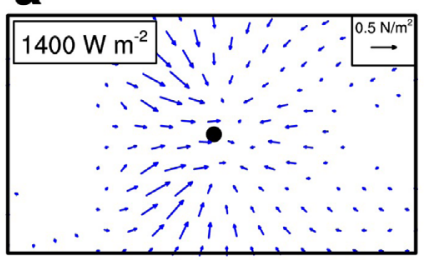

e

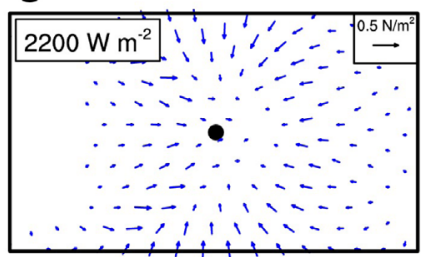

i

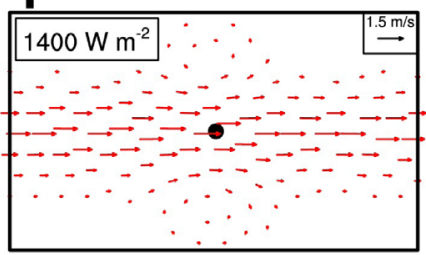

m

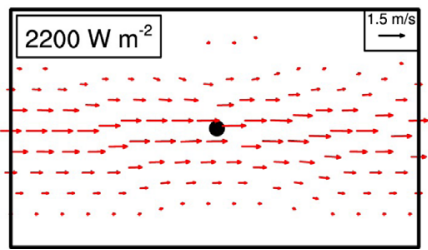

b

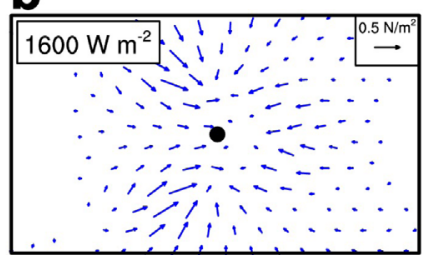

$f$

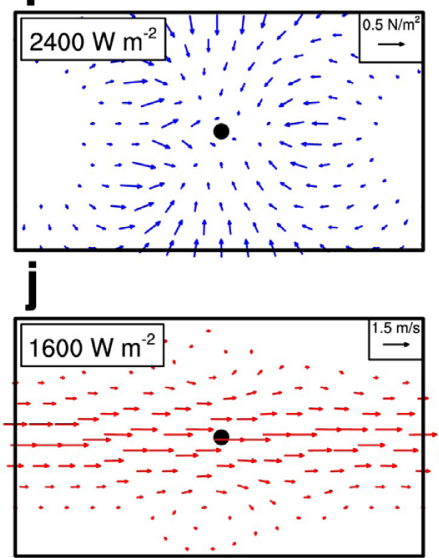

n

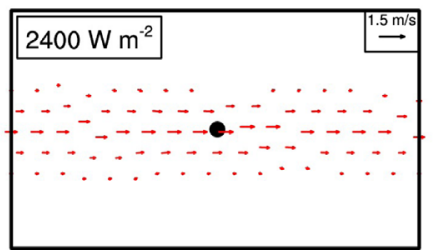

C

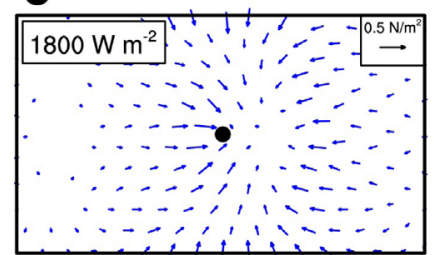

g

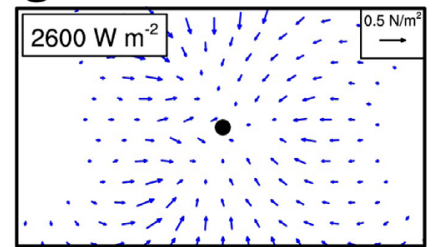

$k$

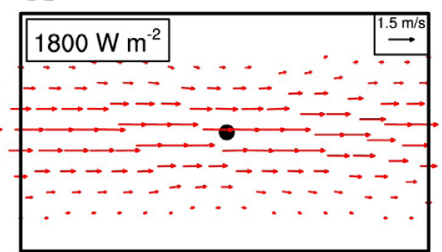

0

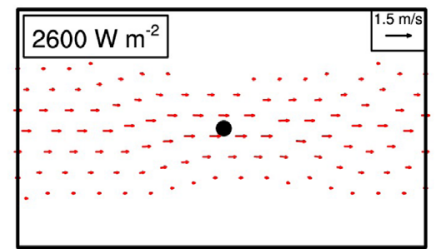

d

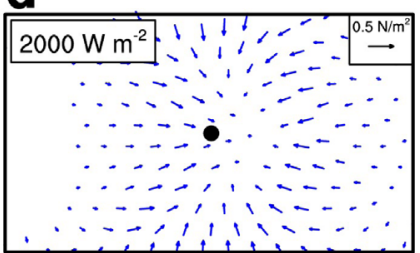

$h$

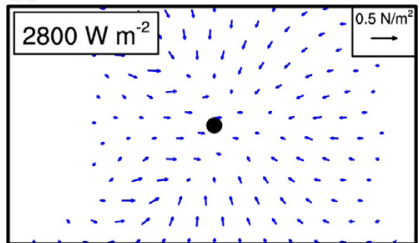

I

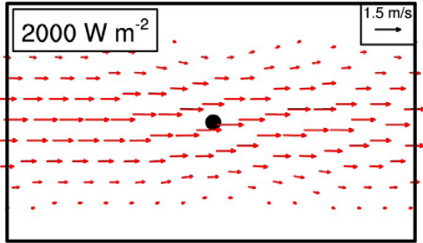

p

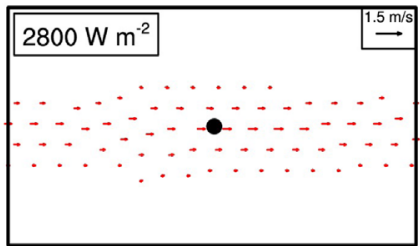

Figure 5. The first reason for the weakening trend of ocean heat transport with increasing stellar flux: wind stresses weaken. Surface wind stresses on the ocean (a)-(f) and vertically averaged ocean currents (g)-(1) on tidally locked aqua-planets, as simulated by CCSM3. The stellar fluxes are 1400, 1600, 1800, 2000, 2200, 2400, 2600 , and $2800 \mathrm{~W} \mathrm{~m}^{-2}$. The horizontal axis is longitude from $0^{\circ}$ to $360^{\circ}$, and the vertical axis is latitude from $85^{\circ} \mathrm{S}$ to $85^{\circ} \mathrm{N}$. The black dot is the substellar point. The reference vector for stresses is $0.5 \mathrm{~N} \mathrm{~m}^{-2}$, and values less than $0.05 \mathrm{~N} \mathrm{~m}^{-2}$ are not plotted. The reference vector for ocean velocity is $1.5 \mathrm{~m} \mathrm{~s} \mathrm{~s}^{-1}$, and values less than $0.15 \mathrm{~m} \mathrm{~s}^{-1}$ are not plotted.

Y. Zeng et al. 2018, in preparation). Surface temperature gradients on the east side of the substellar point are smaller than those on its west side (see the right panel of Figure 2(b)). Due to this asymmetry, the eastward stresses on the west side of the substellar point are generally stronger than the westward stresses on the east side of the substellar point (Figures 5(a)-(h)), so the net effect of the surface stresses is to drive eastward ocean flows. As the stellar flux is increased, the ocean flows become weaker (Figures 5(i)(p)) due to weaker surface wind stresses (Figures 4(h) and 5(a)(h)). The decrease in surface wind stresses at least partly results from a smaller surface temperature gradient (Figure 4(g)), which is associated with the greater warming of the nightside compared to the dayside. ${ }^{7}$

The ocean currents become ineffective when the stellar flux is equal to $2400 \mathrm{~W} \mathrm{~m}^{-2}$ or higher (Figures $5(\mathrm{n})-(\mathrm{p})$ ). Our

\footnotetext{
7 Note that the surface temperature gradient in CCSM3 is much smaller than that in CAM3; this is due to the effect of ocean heat transport in the coupled model and associated feedback processes (see Section 3.1). Moreover, the surface temperature gradient is weak in all of CCSM3's experiments except for stellar fluxes of 1400 and $1600 \mathrm{~W} \mathrm{~m}^{-2}$, but the surface wind stress generally decreases with increasing stellar flux (Figures 4(f)-(h)). This implies that surface temperature gradient is not the only determinant of the strength of surface wind stress; other factors, such as downward momentum flux from the free troposphere to the surface (chapter 12 of Vallis 2006), may be very important in certain conditions; and future work is required to fully understand this.
}

finding that small horizontal temperature gradients and weak surface wind stresses when the stellar flux is high is compatible with previous work on hot climates under high levels of insolation or atmospheric $\mathrm{CO}_{2}$. Examples of this are shown in Figure 1 of Leconte et al. (2013a) for the climate simulation of Earth under a brightening Sun, in Figure 2 of Way et al. (2016) for a temperate Venus, and in Figure 4 of Wolf (2017) for the exoplanet TRAPPIST $1 \mathrm{~d}$.

As the stellar flux is increased, both shortwave absorption by water vapor (Figure 6(a)) and shortwave reflection by clouds increase (Figure 4(i)). The increase in shortwave absorption by water vapor is primarily due to the increase in saturation vapor pressure with temperature following the Clausius-Clapeyron relation (Figure 6(b)). The increase of shortwave reflection by clouds is due to the effect of a stabilizing cloud feedback: greater stellar flux produces stronger substellar convection, more optically thick clouds, and a higher planetary albedo (Yang et al. 2013). This phenomenon was first found in atmosphere-only general circulation models and briefly tested in CCSM3; here we confirm that this feedback exists in a broader range of coupled ocean-atmosphere simulations. Both the increased water vapor absorption and enhanced cloud reflection lead to the surprising result that the stellar energy reaching the sea surface actually decreases as the stellar flux at the top of the atmosphere is increased (Figure 6(c)). 

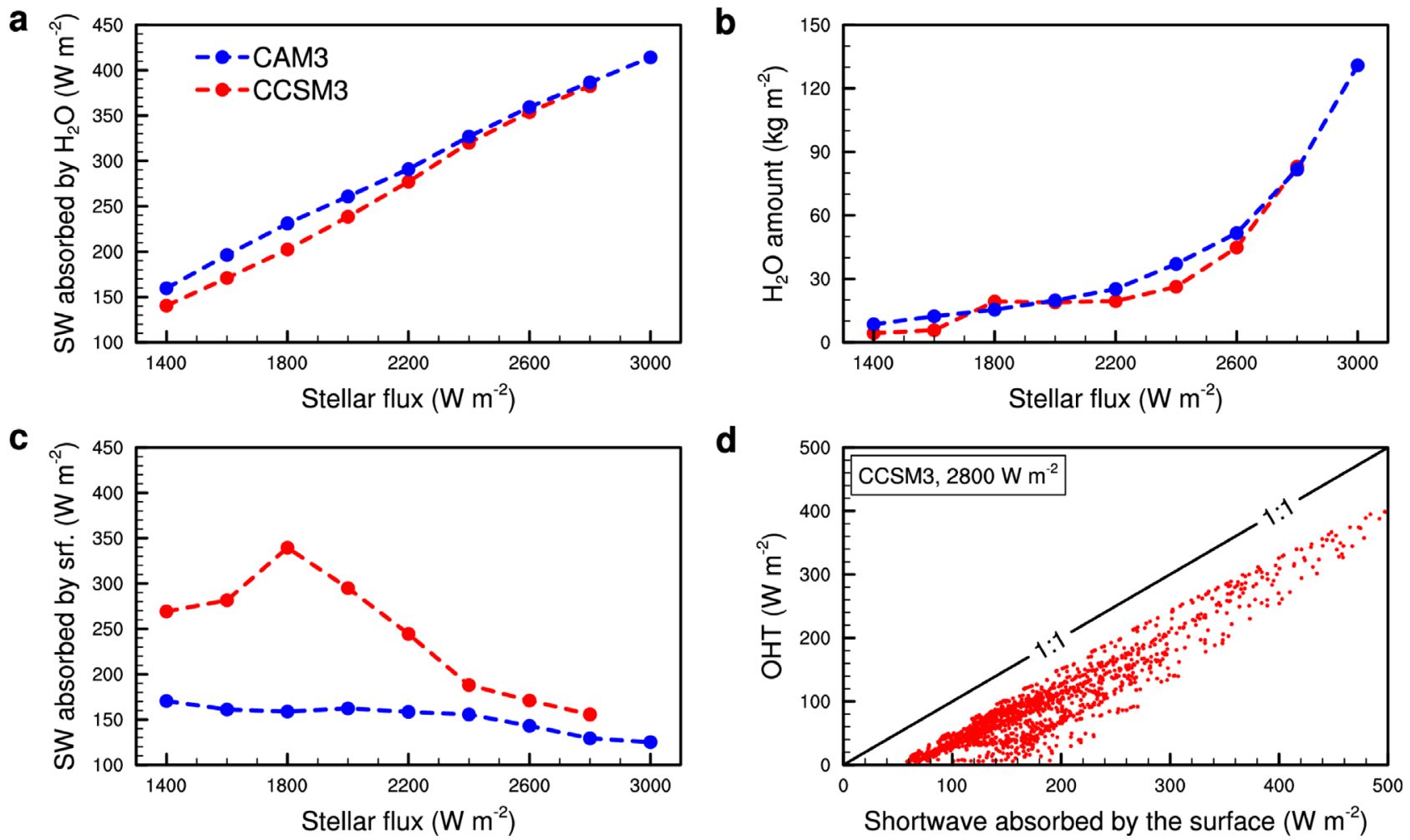

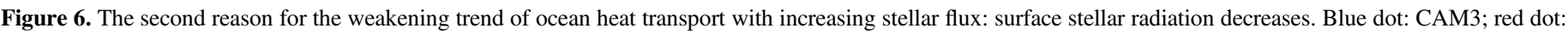

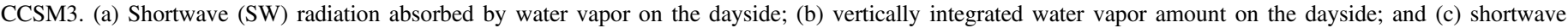

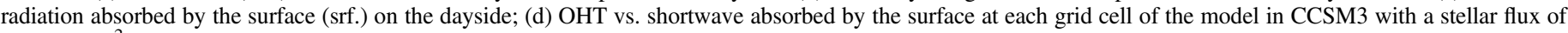

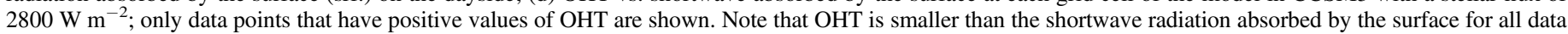
points; the cases of other stellar fluxes have the same relationship (figure not shown).

For long-time mean climatology, the surface energy balance of an ice-free region can be written as: $\mathrm{OHT}=R_{s}-R_{l}-\mathrm{SH}-\mathrm{LH}$, where $R_{s}$ is net shortwave radiation flux into the surface, $R_{l}$ is net longwave radiation flux from the surface to the atmosphere, $\mathrm{SH}$ is sensible heat flux from the surface to the atmosphere, and LH is latent heat flux from the surface to the atmosphere. All of these variables are positive as long as there is no temperature inversion (under an inversion, $\mathrm{SH}$ and $R_{l}$ will be negative). This equation implies that $\mathrm{OHT}<R_{s}$, i.e., the maximum allowed $\mathrm{OHT}$, is constrained by the absorbed shortwave radiation at the sea surface, as shown in Figure 6(d). The value of $R_{s}$ decreases with increasing stellar flux (Figure 6(c)), so that OHT should have the same trend.

Note that although the value of $R_{s}$ decreases with stellar flux, the surface temperatures generally keep increasing (Figure 4(d)(e)); this is mainly due to the increasing of the atmospheric greenhouse effect. The physical process can be briefly summarized as follows: when the stellar flux is increased, more shortwave radiation is absorbed by water vapor (Figure 6(a)), which acts to increase the air temperature and thereby the atmosphere is able to hold more water vapor based on the Clausius-Clapeyron relation. The increased water vapor concentration raises the greenhouse effect of the atmosphere (Figure 7(d)) and emits more longwave radiation to the surface (Figure 7(a)) such that the net longwave radiation at the surface decreases with stellar flux (Figure 7(c)), warming the surface. Moreover, as the stellar flux is increased, the surface sensible heat flux generally decreases (Figure 7(f)), which has a secondary warming effect on the surface.

The day-to-night OHT decreases with increasing stellar flux because of the above two mechanisms, weaker surface wind stresses, and less stellar energy deposited at the surface. In the $1400 \mathrm{~W} \mathrm{~m}^{-2}$ simulation, the OHT is $124 \mathrm{~W} \mathrm{~m}^{-2}$, which contributes to $63 \%$ of the total heat transport, whereas in the $2800 \mathrm{~W} \mathrm{~m}^{-2}$ simulation, it decreases to $70 \mathrm{~W} \mathrm{~m}^{-2}$ and the percentage reduces to $23 \%$ (Figure 4(c)). These results indicate that at the inner edge of the habitable zone, the OHT would be even smaller, although the total (atmosphere plus ocean) heat transport would still be very robust.

The decreasing trend of OHT with increasing stellar flux suggests that ocean dynamics may be not important for very hot climates. Indeed, we find that the location of the inner edge of the habitable zone does not depend on ocean dynamics (Figure 8). For a stellar temperature of $4500 \mathrm{~K}$, the climate system enters a moist greenhouse state at a stellar flux of $\simeq 2600-2800 \mathrm{~W} \mathrm{~m}^{-2}$ for both CCSM3 and CAM3 (Figure 8(a)). At this level of stellar flux, the stratospheric water vapor concentration, $\geqslant 3000 \mathrm{ppmv}$, is high enough that $\mathrm{H}_{2} \mathrm{O}$ photodissociation and subsequent $\mathrm{H}$ escape to space becomes significant, which is known as the moist greenhouse state (Kasting 1988; Kasting et al. 1993). It should be mentioned that at a stellar flux of $2600 \mathrm{~W} \mathrm{~m}^{-2}$, the day-to-night OHT is still about $25 \%$ of the total heat transport and the difference in global-mean surface temperature between CCSM3 and CAM3 is still up to $25 \mathrm{~K}$. However, the key variable for the onset of the moist greenhouse state is the stratospheric water vapor concentration rather than the global-mean surface temperature or other variable(s).

For lower stellar fluxes, stratospheric water vapor in CCSM3 is slightly lower than that in CAM3 (Figure 8(a)), due to relatively weaker vertical velocities in the stratosphere over the substellar region in CCSM3 than those in CAM3 (figure not shown). At higher stellar flux, however, the day-to-night OHT become weaker and the two models have the same concentration of stratospheric water vapor. On tidally locked planets, the 

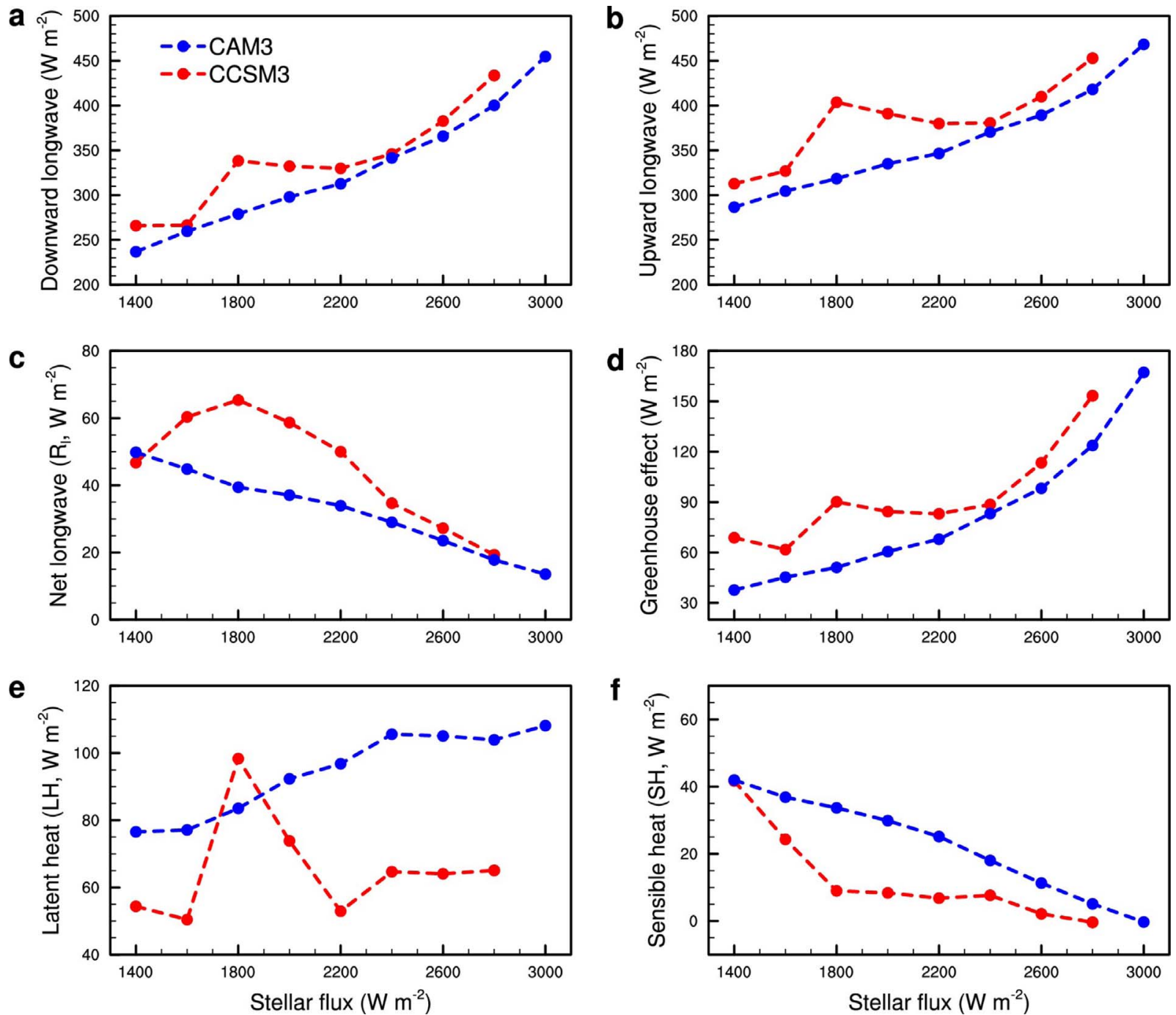

Figure 7. Surface energy flux and greenhouse effect as a function of stellar flux. (a) Downward longwave radiation flux at the surface; (b) upward longwave radiation flux at the surface; (c) net longwave radiation flux (upward minus downward); (d) clear-sky greenhouse effect, defined as $\sigma T_{s}^{4}-\mathrm{OLR}_{\text {clear }}$, where $T_{s}$ is the surface temperature, OLR $_{\text {clear }}$ is the clear-sky outgoing longwave radiation at the top of the model, and $\sigma$ is the Stefan-Boltzmann constant (Pierrehumbert 2005); (e) latent heat flux at the surface; and (f) sensible heat flux at the surface. All of these variables are area averages on the dayside only.
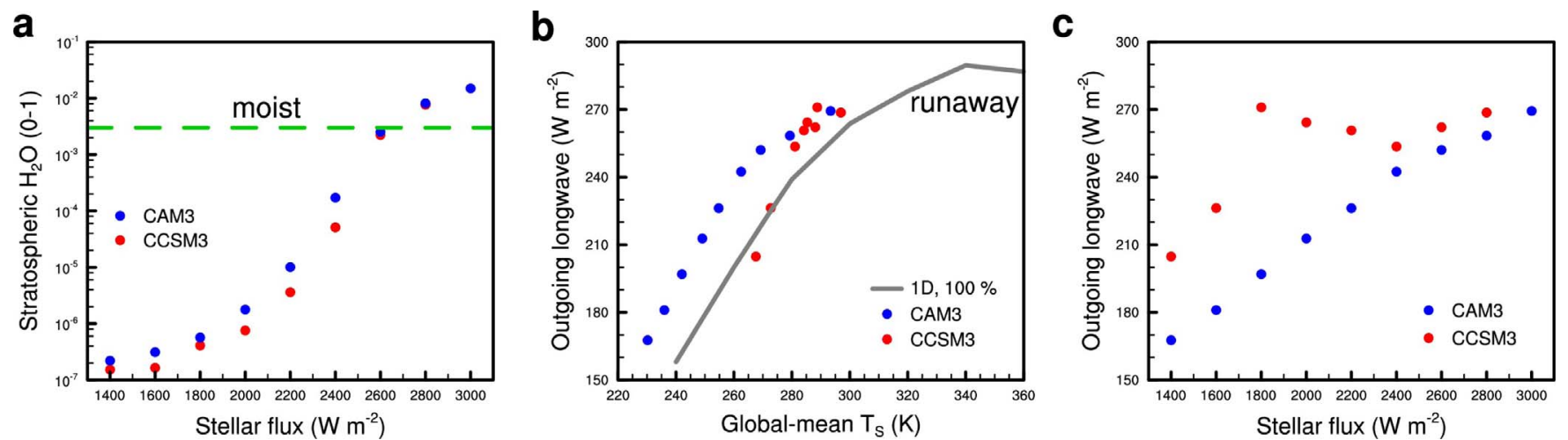

Figure 8. The effect of ocean dynamics on the inner edge of the habitable zone for a tidally locked aqua-planet around a low-mass star. Red dots: CCSM3; blue dots: CAM3. (a) Global-mean stratospheric water vapor mixing ratio at $50 \mathrm{hPa}$, as a function of stellar flux (horizontal green line: the moist greenhouse limit of $3000 \mathrm{ppmv}$ ). (b) Global-mean outgoing longwave radiation (OLR) as a function of surface temperature. The gray line in (b) is the OLR from a 1D cloud-free radiative transfer model assuming $100 \%$ relative humidity, as calculated by Leconte et al. (2013a). (c) OLR as a function of stellar flux.

stratospheric water vapor abundance is primarily determined by the temperature of the tropopause and the strength of the stratospheric vertical velocity above the substellar region. The vertical velocity is mainly driven by near-infrared radiative heating associated with shortwave absorption by stratospheric water vapor and cloud particles (Fujii et al. 2017). 
Finally, our results suggest that the ocean-atmosphere and atmosphere-only models would enter into a runaway greenhouse state, in which absorbed shortwave exceeds maximum allowed outgoing longwave $\left(\mathrm{OLR}_{\max }\right)$, at a similar stellar flux. The stellar flux limit $\left(S_{\text {limit }}\right)$ for triggering the runaway greenhouse only depends on OLR $\mathrm{Rax}_{\text {ax }}$ and the planetary albedo near the inner edge $\left(\alpha_{p}\right)$, i.e., $S_{\text {limit }}=4 \times \operatorname{OLR}_{\max } /\left(1-\alpha_{p}\right)$, where the factor 4 is the ratio of a spheres surface area to its cross-sectional area. Figure 8(b) shows that the two models likely have the same OLR $\mathrm{max}_{\text {man }}$ Meanwhile, they exhibit nearly the same $\alpha_{p}$ as the stellar flux is increased to $2800 \mathrm{~W} \mathrm{~m}^{-2}$ (Figure 4(i)), although the global-mean surface temperature still has a difference of $20 \mathrm{~K}$ and the day-to-night OHT is still $23 \%$ of the total heat transport. In the models we used, the stellar flux limit ${ }^{8}$ is about $3300 \mathrm{~W} \mathrm{~m}^{-2}$ both with and without ocean dynamics. This limit is about two times that for rapidly rotating planets around $\mathrm{G}$ stars (such as Earth, Leconte et al. 2013a), primarily due to the stabilizing cloud feedback (Yang et al. 2013). In summary, ocean dynamics do not influence the stellar flux limit for the onset of the runaway greenhouse state in our experiments using a 60 day tidally locked orbit.

As shown in Figure 8(b), for lower stellar fluxes and under the same global-mean surface temperature, outgoing longwave radiation at the top of the model in CAM3 is higher than that in CCSM3. This is due to the absence of a nightside temperature inversion (Figure 2(f)) and the stronger longwave cloud radiative effect in CCSM3 (Figure 3), both of which reduce the outgoing longwave radiation in the coupled atmosphereocean model. When the stellar flux is $\geqslant 3000 \mathrm{~W} \mathrm{~m}^{-2}$, the temperature inversion in CAM3 disappears and the longwave cloud radiative effect becomes close to that of CCSM3 (Figure 3(b)), so that the outgoing longwave radiation fluxes of the two models coverage on each other (Figure 8(b)). Note that due to numerical instability, CCSM3 blew up for stellar fluxes higher than $2800 \mathrm{~W} \mathrm{~m}^{-2}$, so the maximum allowed outgoing longwave radiation cannot be read from Figure 8(b). However, our present experiments show no evidence that ocean dynamics affects the maximum allowed outgoing longwave radiation and the planetary albedo at the inner edge of the habitable zone.

Another way to understand the differences and similarities between CAM3 and CCSM3 is by plotting the global-mean outgoing longwave radiation as a function of the stellar radiation downward at the top of the atmosphere, as shown in Figure 8(c). At equilibrium, outgoing longwave radiation is equal to absorbed stellar radiation. At lower stellar fluxes, the outgoing longwave radiation is higher in CCSM3 than in CAM3 because CCSM3 has a lower planetary albedo (see Figure 4(i)) and therefore absorbs more stellar radiation. For a high stellar flux, $\geqslant 2400 \mathrm{~W} \mathrm{~m}^{-2}$, the difference in the outgoing

\footnotetext{
There is a significant uncertainty in $\mathrm{OLR}_{\max }$ (as well as in $\alpha_{p}$ ), $295 \pm 15 \mathrm{~W} \mathrm{~m}^{-2}$, arising from clouds, the degree of atmospheric subsaturation, and the uncertainties in radiative transfer calculations (Leconte et al. 2013a; Wolf \& Toon 2015; Yang et al. 2016; Marcq et al. 2017). For a star with a temperature of $4500 \mathrm{~K}$, the value of $\alpha_{p}$ is $\simeq 0.62$ (Figure 4 (f)), and therefore the stellar flux limit is about $3100 \pm 160 \mathrm{~W} \mathrm{~m}^{-2}$. If we further assume a $10 \%$ uncertainty (it could be even larger because clouds are not explicitly resolved and models employ different cloud parameterization schemes) in $\alpha_{p}$, the stellar flux limit would be about $3100 \pm 650 \mathrm{~W} \mathrm{~m}^{-2}$. For the models used in this study, the maximum allowed clear-sky outgoing longwave radiation is about $355 \mathrm{~W} \mathrm{~m}^{-2}$ (see Figure 7(c) in Yang et al. 2018), the cloud longwave radiative effect is about $45 \mathrm{~W} \mathrm{~m}^{-2}$ (Figure $3(\mathrm{a})$ ), and the value of $\alpha_{p}$ is about 0.62 , so that the runaway greenhouse limit is about $3260 \mathrm{~W} \mathrm{~m}^{-2}$. Our last converged experiment, $2800 \mathrm{~W} \mathrm{~m}^{-2}$, is still $460 \mathrm{~W} \mathrm{~m}^{-2}$ less than this limit.
}

longwave radiation between CAM3 and CCSM3 becomes small; this again suggests that ocean dynamics likely have no significant effect on the inner edge of the habitable zone.

\subsection{Observational Thermal Phase Curves}

We further find that ocean dynamics have a very small effect on the observational thermal phase curve of tidally locked planets near the inner edge of the habitable zone. Figure 9 shows the spatial pattern of thermal emission at the top of the atmosphere and thermal phase curves. The phase curves are disk-integrated thermal radiation measured by an observer as a function of the planet's position in its orbit (e.g., Cowan \& Agol 2008; Koll \& Abbot 2015). The curves are determined by the combined effect of surface temperature, water vapor, clouds, and atmospheric heat transport and OHT; therefore, they can be used to probe the atmosphere and/or surface characteristics of exoplanets.

For planets near the inner edge of the habitable zone, the day-to-night OHT is not strong, so the phase curves of CAM3 and CCSM3 are similar (Figure 9(f)). Importantly, the thermal emission on the nightside is much higher than that on the dayside, reversing the day-night thermal contrast (Figures 9(b), (d)). This reversal is due to the high concentration of water vapor and clouds above the substellar region, which absorb the thermal radiation from the surface but re-emit to space at much lower temperatures (Yang et al. 2013; Haqq-Misra et al. 2017). Meanwhile, the nightside is relatively drier and therefore infrared radiation from near the surface can be lost to space easily (Pierrehumbert 1995; Yang \& Abbot 2014). In CAM3, the phase curve reversal occurs when the stellar flux is equal to or higher than $2200 \mathrm{~W} \mathrm{~m}^{-2}$, whereas in CCSM3 it occurs at a much lower stellar flux, $1600 \mathrm{~W} \mathrm{~m}^{-2}$ (Figures $9(\mathrm{~g})$, (h)). This suggests that planets with deep oceans in the middle range of the habitable zone could also have higher thermal emission on the nightside than on the dayside.

For a stellar flux of $1400 \mathrm{~W} \mathrm{~m}^{-2}$ in CAM3, the phase curve has a maximum when the observer sees the dayside and a minimum when the observer views the nightside (Figure 9(e)). When ocean dynamics are included, more heat is transported to the nightside, so the day-night thermal contrast in CCSM3 is much smaller than that in CAM3 and therefore the amplitude of the phase curve is much smaller. Furthermore, because most of the heat is transported to the east side (rather than the west side) of the substellar point (Figure 9(c)), the ridge of the phase curve exhibits a positive phase angle displacement of $\simeq 120^{\circ}$ (Figure 9(e)). In contrast, when ocean dynamics are not considered, the thermal emission on the west side of the substellar point is relatively higher than that on the east side (Figure 9(a)), because water vapor is transported eastward, where it absorbs thermal emission from the surface, and therefore the thermal phase curve exhibits a small negative phase angle displacement (Figure 9(e), see also Figure 3(b) in Yang et al. 2013).

Could the oceanic effect on the thermal phase curve be observed by the James Webb Space Telescope (JWST)? To estimate JWST's precision, we assume that observations are only limited by photon noise and by the telescope's detector efficiency (see Koll \& Abbot 2015). For the target we assume the LHS 1140 system (Dittmann et al. 2017); closer or brighter host stars would be easier to measure. For the instrument we assume MIRIF1800W (16.5-19.5 $\mu \mathrm{m})$ photometry and a photon efficiency of $1 / 3$. Because habitable zone planets are cool, it is necessary to 

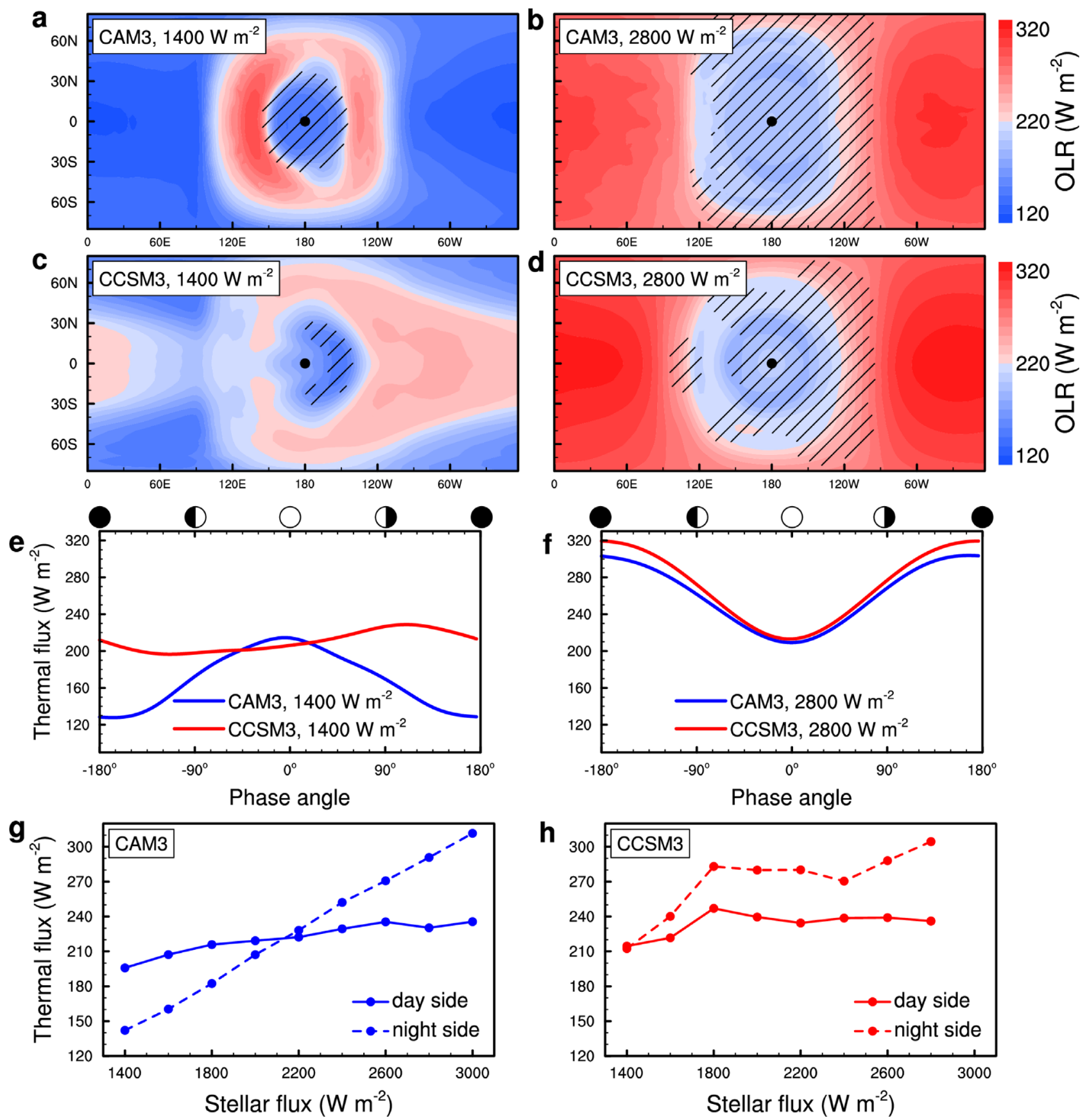

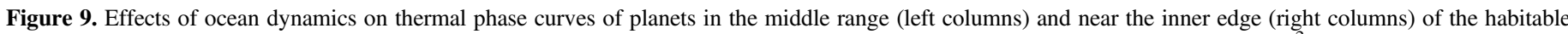

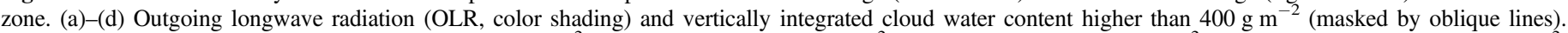

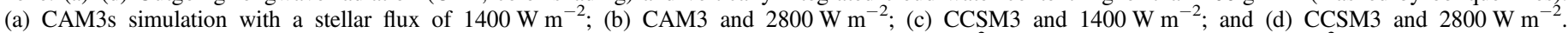

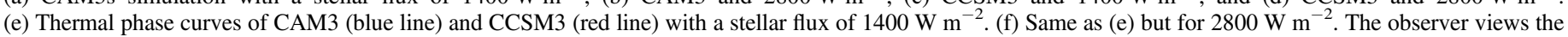

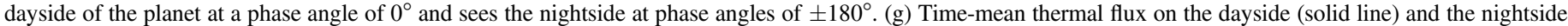

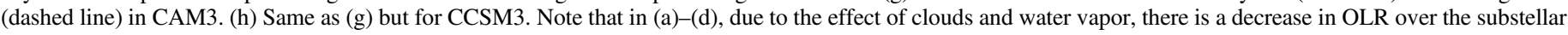
point in all experiments.

observe at long wavelengths to obtain favorable planet-star thermal contrasts. We note that at such long wavelengths additional sources of error could become significant, e.g., dust or thermal background, so our estimate is optimistic. For a $2 \mathrm{hr}$ integration the $1 \sigma$ error bar for the flux will be $144 \mathrm{~W} \mathrm{~m}^{-2}$. For a $24 \mathrm{hr}$ integration, the error goes down by $1 / \sqrt{12}$, so a $1 \sigma$ error would be $\simeq 42 \mathrm{~W} \mathrm{~m}^{-2}$, larger than the amplitude of the thermal phase curve shift under ocean dynamics in the middle of the habitable zone (see the red line in Figure 9(e)) but smaller than the amplitude of the day-night phase curve reversal near the inner edge of the habitable zone (Figure 9(f)). Therefore, JWST observations with long staring exposures would be able to detect the thermal phase curve reversal near the inner edge of the habitable zone. However, unless future discoveries detect a target more favorable than LHS 1140b, the ocean-induced thermal phase shift would likely not be detectable with JWST. 


\subsection{Non-monotonic Behavior of the Coupled Atmosphere- Ocean System}

As shown in Figures 3, 4, 6, and 7, the climate in CCSM3 is not a monotonic function of stellar flux. Variables that show the non-monotonic behavior include: (1) the day-to-night ocean heat transport (Figure 4(a)), nightside longwave cloud radiative effect (Figure 3(b)), dayside shortwave absorption by the sea surface (Figure 6(c)), and surface net longwave radiation (Figure 7(c)) increase with stellar flux between 1400 and $1800 \mathrm{~W} \mathrm{~m}^{-2}$ but decrease with stellar flux when it is higher than $1800 \mathrm{~W} \mathrm{~m}^{-2}$; (2) the global-mean surface temperature (Figure 4(d)), day-tonight total heat transport (Figure 4(b)), global-mean longwave cloud radiative effect (Figure 3(a)), and both upward and downward longwave radiation fluxes at the surface (Figures 7(a) and (b)) increase between 1400 and $1800 \mathrm{~W} \mathrm{~m}^{-2}$, decrease between 1800 and $2400 \mathrm{~W} \mathrm{~m}^{-2}$, and increase again when the stellar flux is higher than $2400 \mathrm{~W} \mathrm{~m}^{-2}$; (3) the planetary albedo (Figure 4(i)) mostly increases with stellar flux but has a minimum value when the stellar flux is $1800 \mathrm{~W} \mathrm{~m}^{-2}$. Sensitivity tests show that this non-monotonic behavior seems to not depend on the initial state (Figures 1(e)-(f)).

The non-monotonic behavior in CCSM3 likely results from atmosphere-ocean interactions and associated feedback processes because the climate simulated using the atmosphere-only model CAM3 is close to monotonic (see Figures 3, 4,6 , and 7). The increase in global-mean surface temperature between 1400 and $1600 \mathrm{~W} \mathrm{~m}^{-2}$ and between 2400 and $2800 \mathrm{~W} \mathrm{~m}^{-2}$ in CCSM3 is relatively easier to understand, whereas the slight decrease in global-mean surface temperature between 1800 and $2200 \mathrm{~W} \mathrm{~m}^{-2}$ and the minimum in planetary albedo at $1800 \mathrm{~W} \mathrm{~m}^{-2}$ are due to the complex interactions between the ocean, atmosphere, and clouds. Comparing the 1400 and $1600 \mathrm{~W} \mathrm{~m}^{-2}$ cases, the nightside is covered by sea ice in both experiments (Figure 10(b)) and the surface temperature gradients decrease, but not very significantly (Figures 10(a) and 4(f)-(g)), so the OHT increases with increasing stellar flux. This implies that in these two experiments of relatively lower stellar flux the two mechanisms - the surface temperature gradient decreasing and the surface wind stress weakening addressed in Section 3.2 are not active enough to counteract the effect of increasing stellar flux. In the 2400,2600 , and $2800 \mathrm{~W} \mathrm{~m}^{-2}$ experiments, the two mechanisms are very effective in reducing the day-to-night OHT although the stellar flux is increased and the atmospheric heat transport increases with stellar flux; as shown in Figure 5, the ocean currents in these three cases are much weaker than those in all other experiments. For the cases between 1800 and $2200 \mathrm{~W} \mathrm{~m}^{-2}$, the situation is more complex and the key may be associated with the effect of ocean dynamics on the spatial pattern of sea surface temperature and consequently on the atmospheric heating rate, the strength of atmospheric super-rotation (winds blowing from west to east over the deep-tropical region) and the spatial pattern of clouds (Figure 10). In the $1800 \mathrm{~W} \mathrm{~m}^{-2}$ case of CCSM3, strong ocean currents transport relatively cold seawater from the nightside to the west side of the substellar point and also transport relatively warm seawater from the substellar point to its east side (similar behavior also occurs at 2000 and $2200 \mathrm{~W} \mathrm{~m}^{-2}$, but the $1800 \mathrm{~W} \mathrm{~m}^{-2}$ case is the most significant). As a result, the sea surface temperature exhibits a strong, zonally asymmetric pattern with the east side of the substellar point being much warmer than the west side (Figure 10(a)). This asymmetric pattern causes the water vapor concentration and atmospheric heating rate to show a similar zonally asymmetric pattern (Figures 10(c)-(d)). The asymmetric heating pattern is likely more effective at generating Rossby waves and Kelvin waves in the atmosphere; the waves pump eastward momentum from higher latitudes to the equator (Showman \& Polvani 2011), inducing very strong super-rotating winds over the substellar region (Figures 10(e) and (j)); these winds advect clouds eastward away from the substellar region where the stellar flux peaks, and the west side of the substellar point becomes nearly cloud-free (Figure 10(f)); as a result, the planetary albedo becomes smaller $\left(0.39\right.$ in the case of $1800 \mathrm{~W} \mathrm{~m}^{-2}$ versus 0.44 in the case of $1600 \mathrm{~W} \mathrm{~m}^{-2}$, suggesting that the stabilizing cloud feedback is less active for this case); therefore, more stellar radiation reaches the surface, warming the sea surface. In CAM3, this phenomenon does not occur because the dayside sea surface temperature is nearly symmetric around the substellar point and the atmospheric zonal winds are weak in all of the experiments (Figure 11).

Moreover, in CCSM3 the strength of the atmospheric superrotation decreases with stellar flux between 1800 and $2200 \mathrm{~W} \mathrm{~m}^{-2}$ (Figure $10(\mathrm{j})$ ), so that the planetary albedo increases with stellar flux, which enhances the primary effect of the stabilizing cloud feedback with increasing stellar flux (Yang et al. 2013). The planetary albedo increases with stellar flux fastest in these three experiments (Figure 4(i)) and as a result the global-mean surface temperature decreases slightly (rather than increases) with stellar flux (Figure 4(d)). The decrease in OHT of these three experiments is due to the combined effect of the planetary albedo increasing (so less surface shortwave radiation is deposited) and surface wind stress weakening (Figures 4(h) and 5). Further work is required to clearly understand the onset condition for strong atmospheric super-rotation in the coupled model, especially as relates to the role of the spatial pattern of surface temperature or atmospheric heating.

Overall, the non-monotonicity of CCSM3 is likely due to complex interactions among the sea surface, atmospheric circulation, water vapor, and clouds. This further demonstrates why fully coupled atmosphere-ocean modeling is required to simulate the details of the climate of planets in the middle of the habitable zone.

\section{Discussion}

An important result of this study is that the location of the habitable zone inner edge should not depend significantly on ocean dynamics. This is consistent with Way et al. (2018), who found that the effect of ocean dynamics on the climate decreases rapidly with stellar flux for a variety of rotation rates in the ROCKE3D GCM (their Figure 2). We should note, however, that our experiments cover a limited range of planetary parameters. We have examined the dynamics of an aqua-planet ocean having a depth of $\simeq 4000 \mathrm{~m}$. Sensitivity tests show that at a stellar flux of $1400 \mathrm{~W} \mathrm{~m}^{-2}$ for shallower oceans or for oceans surrounded by continents, the day-to-night OHT would become smaller and its climatic effect would be weaker (Figure 12). For a shallower ocean, friction at the ocean bottom is more effective at decelerating ocean currents, so less warm substellar seawater can be transported to the cold nightside. In the case of an $800 \mathrm{~m}$ deep ocean, the nightside is much cooler than that in the $4000 \mathrm{~m}$ case as a result of less ocean heat input 


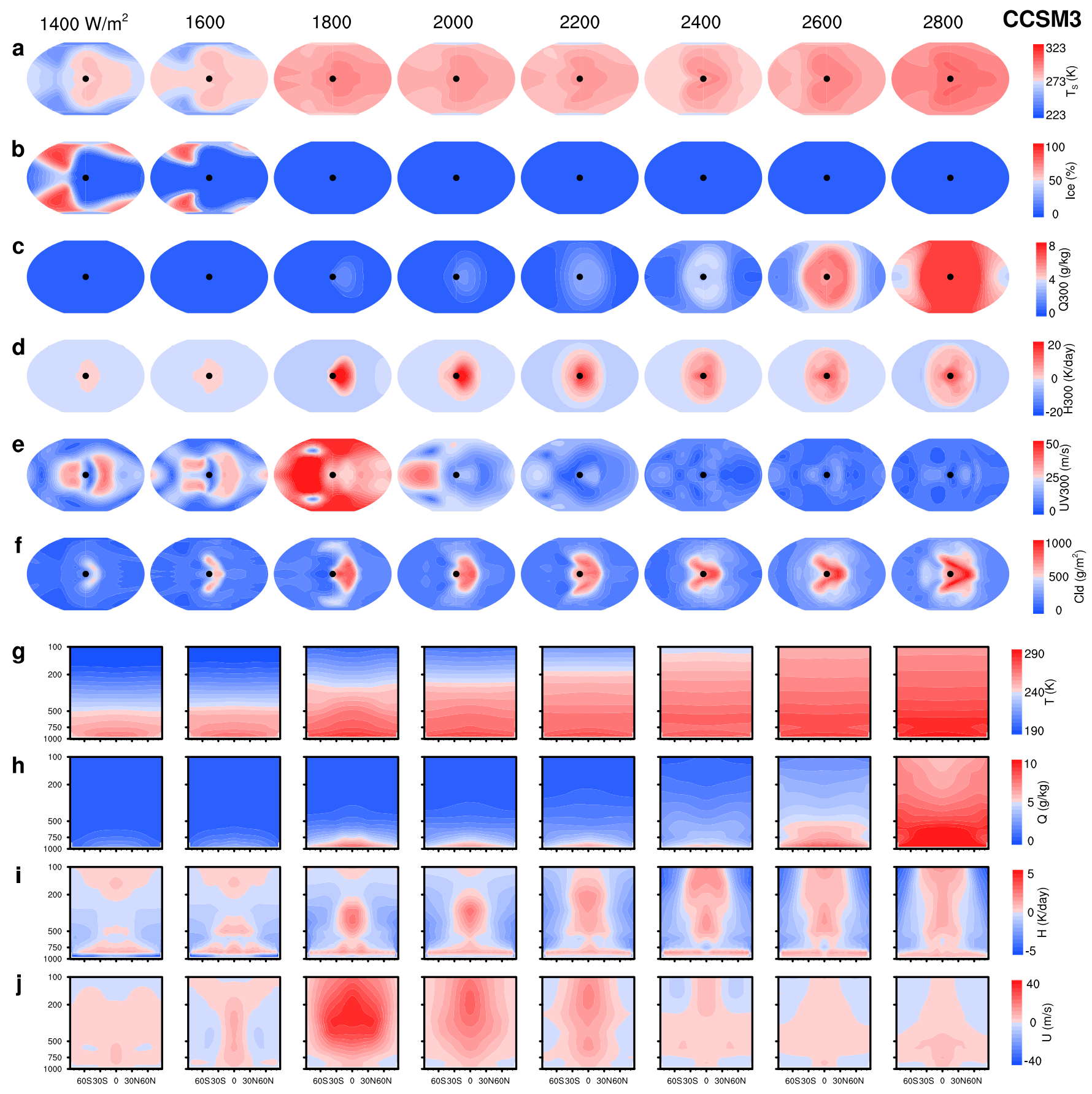

Figure 10. Atmospheric characteristics simulated by CCSM3 at stellar fluxes from 1400 to $2800 \mathrm{~W} \mathrm{~m}^{-2}$ with an interval of $200 \mathrm{~W} \mathrm{~m}^{-2}$. (a) Surface air temperature; (b) sea ice coverage; (c) water vapor specific humidity at $300 \mathrm{hPa}$; (d) heating rate at $300 \mathrm{hPa}$ due to the combined effect of shortwave heating, longwave cooling, and moist processes (such as latent heat release during condensation); (e) horizontal wind strength $\left(\sqrt{u^{2}+v^{2}}\right)$ at $300 \mathrm{hPa}$; (f) vertically integrated cloud water amount; (g) zonal-mean air temperature; (h) zonal-mean water vapor specific humidity; (i) zonal-mean heating rate; and (j) zonal-mean zonal winds. Note that the troposphere and water vapor profiles extend to higher altitudes in a warmer climate, so that more stellar radiation is absorbed by water vapor at high altitudes. The black dot in (a)-(f) denotes the substellar point.

from the dayside. The dayside surface, however, also becomes cooler even though less energy is transported away from the substellar region. This is due to a cloud feedback. Due to the reduced OHT (Figure 12(d)), the surface temperature contrast between the dayside and the nightside increases (Figure 12(c)). The larger surface temperature contrast promotes stronger water vapor convergence above the substellar region, and therefore more clouds form there. These clouds have a strong cooling effect on the sea surface through increasing planetary albedo. The planetary albedos are 0.29 and 0.45 in the cases of 4000 and $800 \mathrm{~m}$, respectively. When the ocean depth is $400 \mathrm{~m}$, the zonal (west-east) ocean currents become weaker (compared to the $800 \mathrm{~m}$ case), so less energy is transported to the nightside through ocean dynamics (Figure 12(f)); however, the meridional (north-south) OHT increases, so the high latitudes become warmer (Figure 12(e)), similar to the results of Del Genio et al. (2019). Compared to the $4000 \mathrm{~m}$ aqua-planet, the trends in the one-barrier case and the two-barrier case are similar to the 


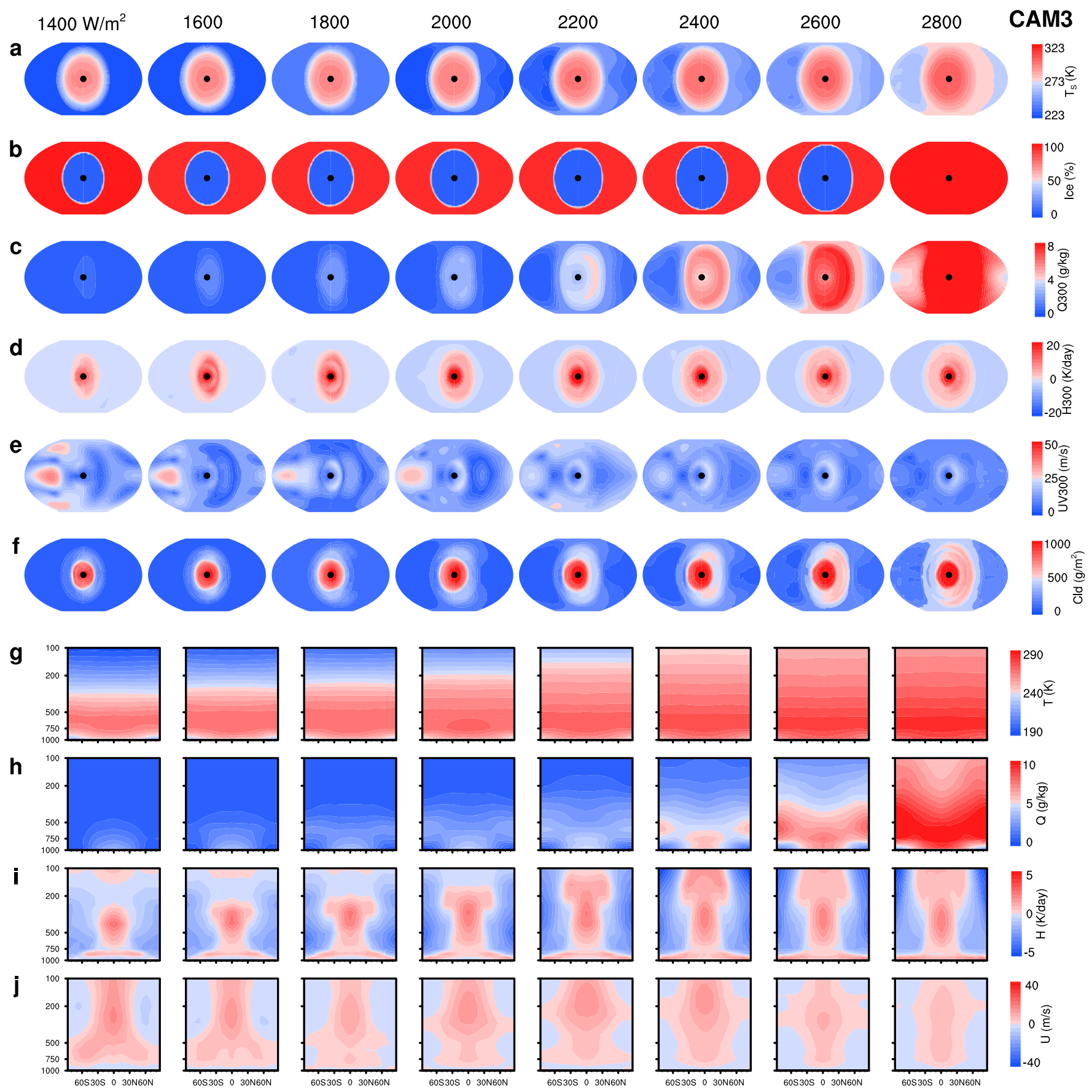

Figure 11. Same as Figure 10 but CAM3 simulations.

$800 \mathrm{~m}$ aqua-planet case: the day-to-night ocean heat transport reduces and both dayside and nightside surfaces become cooler (Figures 12(g)-(h)). In the two-barrier case, the day-to-night OHT is completely blocked by the continents and the ocean currents can transport the substellar heat only to the terminators and to the dayside polar regions, where the surface becomes warmer than that in the one-barrier case (Figures 12(i)-(j)). Future work is required to further investigate the ocean barrier cases, as well as more ocean depth cases, under higher stellar fluxes.

Future work is also required to investigate the effects of ocean dynamics on planets in different spin-orbit resonance states (such as 3:2 for Mercury), on rapidly rotating planets around $\mathrm{G}$ stars (such as Earth) and on planets near the outer edge of the habitable zone, as well as the effects of different atmospheric masses, atmospheric compositions, and deeper oceans (such as ocean worlds, Leger et al. 2003). A higher background atmospheric pressure than the one bar used in this study may further lessen the surface shortwave energy deposition through increasing atmospheric scattering, which may further weaken the effect of ocean dynamics on the inner edge of the habitable zone. A lower background atmospheric pressure should have a small effect on the results shown here because water vapor should dominate atmospheric composition at the inner edge of the habitable zone, especially for the runaway greenhouse state.

In our experiments, the salinity of sea ice is set to $4 \mathrm{~g} \mathrm{~kg}^{-1}$, while the seawater salinity is about $35 \mathrm{~g} \mathrm{~kg}^{-1}$, so that sea ice 

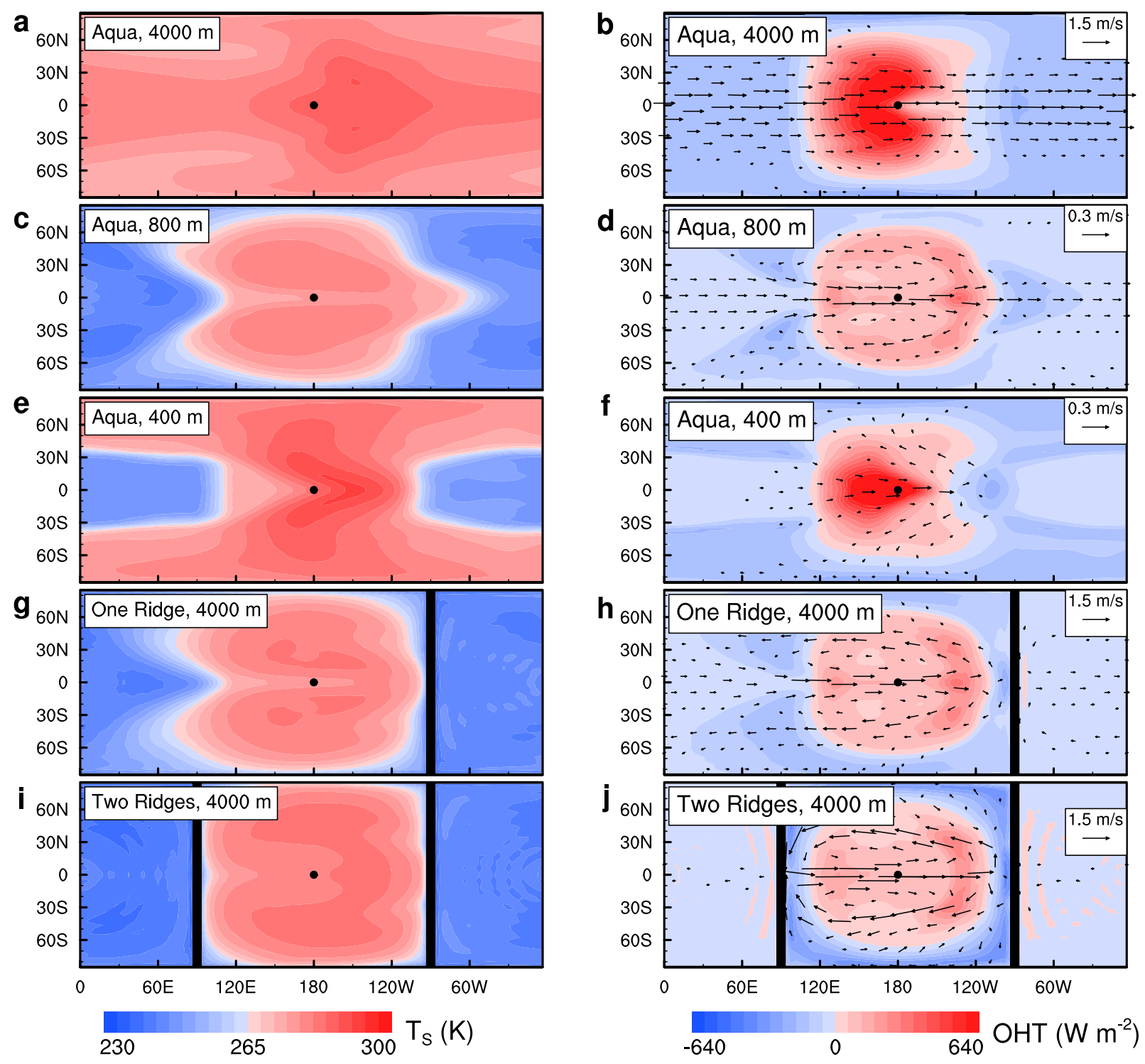

Figure 12. Effects of ocean depth and continental barriers on the long-time mean surface temperature (left panels) and ocean heat transport (OHT, right panels, negative: heat from ocean to atmosphere; positive: heat from atmosphere to ocean). (a) and (b) An aqua-planet with an uniform ocean depth of $4000 \mathrm{~m}$; (c) and (d) an ocean depth of $800 \mathrm{~m}$; (e) and (f) an ocean depth of $400 \mathrm{~m}$; (g) and (h) one-ridge world with an ocean depth of $4000 \mathrm{~m}$; and (i) and (j) two-ridges world with an ocean depth of $4000 \mathrm{~m}$. In the right panels, the vectors denote vertically averaged ocean currents with reference vectors of $1.5,0.3,0.3,1.5$, and $1.5 \mathrm{~m} \mathrm{~s}{ }^{-1}$, respectively. The thick black lines in (g)-(j) denote the continental barriers, which extend from the ocean bottom to the sea surface. The black dot denotes the substellar point. In these simulations, the stellar temperature is $3400 \mathrm{~K}$, the stellar flux is $1400 \mathrm{~W} \mathrm{~m}^{-2}$, and the planetary rotation period is 37 Earth days (see Table 1).

melting and freezing is able to influence the ocean salinity and thereby oceanic thermohaline circulation. The thermohaline circulation has not been shown in this manuscript because the day-to-night OHT is dominated by wind-driven ocean circulations in our experiments. Our model blew up when the stellar flux was higher than $2800 \mathrm{~W} \mathrm{~m}^{-2}$. Future experiments are required for higher stellar fluxes, under which wind-driven ocean circulation would become even weaker and thermohaline circulation might become important. Our preliminary thinking is that for planets near the inner edge of the habitable zone, the thermohaline circulation should be mainly driven by the salinity gradient because the surface temperature gradient is very small. The salinity gradient is mainly determined by the spatial patterns of precipitation and evaporation that are driven by large-scale atmospheric circulation. For planets at the outer edge of the habitable zone, the thermohaline circulation may be larger than that at the inner edge. This is because both temperature and salinity gradients on planets at the outer edge may be strong enough to drive a robust thermohaline circulation. Cullum et al. $(2014,2016)$ examined the effects of planetary rotation and average ocean salinity on the thermohaline circulation using an ocean-only model. Winddriven ocean circulation and the interactions between ocean, atmosphere, and surface climate, however, were not considered in their studies. Future work is required to examine the effect of average ocean salinity on the ocean circulation (as was briefly tested in Del Genio et al. 2019 for Proxima b) and the effect of thermal-driven ocean circulation on the inner and outer edges of the habitable zone.

\section{Summary}

In the middle range of the habitable zone, ocean dynamics significantly warm the nightside and the dayside high latitudes 

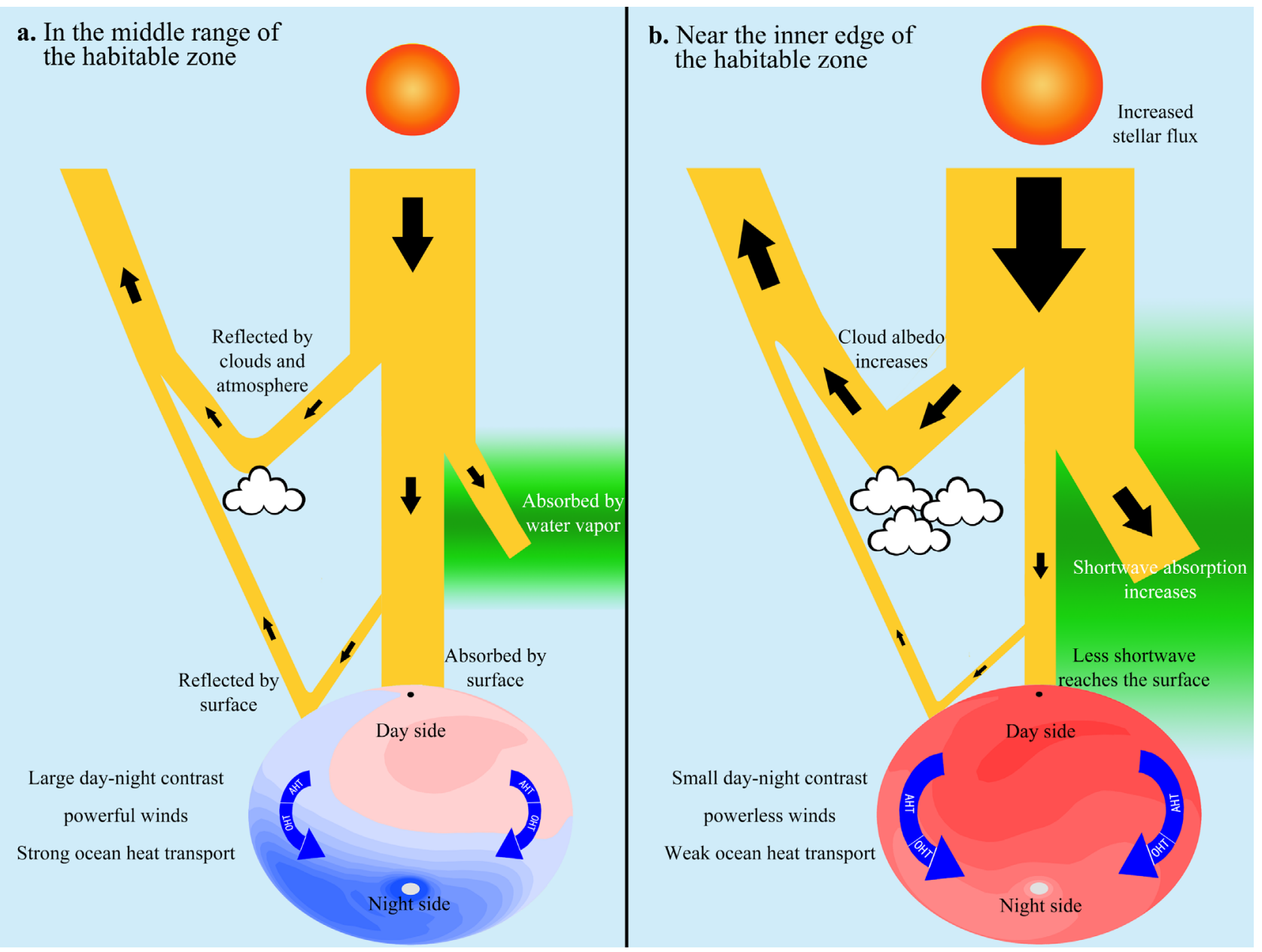

Figure 13. Schematic illustration of the decrease in day-to-night ocean heat transport $(\mathrm{OHT})$ as the stellar flux increases and the underlying mechanisms. The decreasing trend of OHT results from weaker wind stress combined with less stellar radiation depositing energy at the dayside surface. (a) In the middle range of the habitable zone, the day-night surface temperature contrast is strong, the surface wind stresses are robust, stellar radiation absorbed by water vapor or reflected by clouds is relatively small, a large part of the stellar energy reaches the surface, and the ocean dominates the day-to-night heat transport. (b) Near the inner edge of the habitable zone, the surface is hot, the day-night surface temperature contrast is small, the surface wind stresses are weak, stellar radiation absorbed by water vapor or reflected by clouds is relatively large, a small part of the stellar energy reaches the surface, and the atmosphere dominates the day-to-night heat transport.

of 1:1 tidally locked aqua-planets and produce an eastward shift of the hottest point at the surface. For planets near the inner edge of the habitable zone, however, OHT is weak and has nearly no effect on the location of the inner edge or on the thermal phase curves of planets near the inner edge (summarized in Figure 13). The weakening of OHT with increasing stellar flux is due to the combined effect of weakened surface wind stress and decreased surface stellar energy deposition at the sea surface. Atmospheric heat transport increases with stellar flux and dominates on planets near the inner edge of the habitable zone. Finally, we note that the detection of oceans, continents, and atmospheres on distant terrestrial exoplanets is still a big challenge (e.g., Cowan et al. 2009, 2012a, 2012b). Future observations using high temporal frequency specular reflections as well as emission and transmission spectra may be able to infer the surface as well as atmospheric characteristics of nearby transiting planets (Cowan et al. 2015; Greene et al. 2016; Stevenson et al. 2016; Bean et al. 2018).

We are grateful to Junyan Xiong for his help in drawing Figure 13. J.Y. acknowledges support from the National
Science Foundation of China (NSFC) grants 41861124002, 41675071,41606060 , and 41761144072 . Y.H. is supported by NSFC, under grants 41375072 and 41530423.

\section{ORCID iDs}

Jun Yang (i) https://orcid.org/0000-0001-6031-2485

Dorian S. Abbot (10 https://orcid.org/0000-0001-8335-6560

Daniel D. B. Koll (i) https://orcid.org/0000-0002-9076-6901

Yongyun Hu (1) https://orcid.org/0000-0002-4003-4630

\section{References}

Bean, J. L., Stevenson, K. B., Batalha, N. M., et al. 2018, PASP, 130, 11402 Bin, J., Tian, F., \& Liu, L. 2018, E\&PSL, 492, 121

Boutle, I. A., Mayne, N. J., Drummond, B., et al. 2017, A\&A, 601, A120 Briegleb, B., Bitz, C. M., Hunke, E. C., Lipscomb, W. H., \& Schramm, J. L. 2002, Description of the Community Climate System Model Version 2 Sea Ice Model, http://www.cesm.ucar.edu/models/ice-csim

Collins, W. D., Bitz, C. M., Blackmon, M. L., et al. 2006, JCli, 19, 2122

Collins, W. D., Rasch, P. J., Boville, B. A., et al. 2004, Description of the NCAR Community Atmosphere Model (CAM 3.0), http://www.cesm.ucar. edu/models/atm-cam/docs/description/description.pdf

Cowan, N. B., Abbot, D. S., \& Voigt, A. 2012a, ApJL, 752, L3

Cowan, N. B., Abbot, D. S., \& Voigt, A. 2012b, ApJ, 757, 80 
Cowan, N. B., \& Agol, E. 2008, ApJ, 678, 129

Cowan, N. B., Eric, A., Meadows, V. S., et al. 2009, ApJ, 700, 915

Cowan, N. B., Greene, T., Angerhausen, D., et al. 2015, PASP, 127, 311

Cullum, J., Stevens, D. P., \& Joshi, M. 2014, AsBio, 14, 645

Cullum, J., Stevens, D. P., \& Joshi, M. 2016, PNAS, 113, 4278

Del Genio, A. D., Way, M. J., Amundsen, D. S., et al. 2019, AsBio, 19, 99

Dittmann, J. A., Irwin, J. M., Charbonneau, D., et al. 2017, Natur, 544, 333

Edson, A., Lee, S., Bannon, P., Kasting, J. F., \& Pollard, D. 2011, Icar, 212, 1

Enderton, R., \& Vallis, G. K. 2009, JAtS, 66, 1593

Farneti, R., \& Vallis, G. K. 2013, JCli, 26, 7151

Fujii, Y., Genio, D. A. D., \& Amundsen, D. S. 2017, ApJ, 848, 100

Gent, P. R., \& McWilliams, J. C. 1990, JPO, 20, 150

Greene, T. P., Line, M. R., Montero, C., et al. 2016, ApJ, 817, 1

Haqq-Misra, J., Wolf, E. T., Joshi, M., et al. 2017, ApJ, 852, 1

Hartmann, D. 2016, Global Physical Climatology (San Diego, CA: Elsevier), 409

Herweijer, C., Seager, R., Winton, M., \& Clement, A. 2005, Tell, 57, 662

Hu, Y., \& Yang, J. 2014, PNAS, 111, 629

Joshi, M., Haberle, R., \& Reynolds, R. 1997, Icar, 129, 450

Joshi, M. M., \& Haberle, R. M. 2012, AsBio, 12, 3

Kasting, J. F. 1988, Icar, 74, 472

Kasting, J. F., Whitmire, D. P., \& Reynolds, R. T. 1993, Icar, 101, 108

Koll, D. D., \& Abbot, D. S. 2013, JCli, 26, 6742

Koll, D. D. B., \& Abbot, D. S. 2015, ApJ, 802, 21

Kopparapu, R. K., Wolf, E. T., Arney, G., et al. 2017, ApJ, 845, 5

Kopparapu, R. K., Wolf, E. T., Haqq-Misra, J., et al. 2016, ApJ, 819, 84

Large, W. G., McWilliams, J. C., \& Doney, S. C. 1990, JPO, 27, 2418

Leconte, J., Forget, F., Charnay, B., Wordsworth, R., \& Pottier, A. 2013a, Natur, 504, 268

Leconte, J., Forget, F., Charnay, B., et al. 2013b, A\&A, 554, A69

Leger, A., Selsis, F., Sotin, C., et al. 2003, Icar, 169, 499

Liu, Y., Peltier, W., Yang, J., \& Vettoretti, G. 2013, CliPa, 9, 2555

Marcq, E., Salvador, A., Massol, H., \& Davaille, A. 2017, JGRE, 122, 1539

Marshall, J., Ferreira, D., Campin, J. M., \& Enderton, D. 2007, JAtS, 64, 4270

Merlis, T. M., \& Schneider, T. 2010, JAMES, 2, 1

Pierrehumbert, R. T. 1995, JAtS, 52, 1784

Pierrehumbert, R. T. 2005, JGRD, 110, D01111

Pierrehumbert, R. T. 2011, ApJL, 726, L8

Popp, M., Schmidt, H., \& Marotzke, J. 2016, NatCo, 7, 10627
Rosenbloom, N., Shields, C. A., Brady, E., Yeager, S., \& Levis, S. 2011, Using CCSM3 for Paleoclimate Applications, http://opensky.ucar.edu/islandora/ object/technotes\%3A496/datastream/PDF/view

Salameh, J., Popp, M., \& Marotzke, J. 2017, CIDy, 50, 1

Shields, A. L., Meadows, V. S., Bitz, C. M., et al. 2013, AsBio, 13, 715

Showman, A. P., \& Polvani, L. M. 2011, ApJ, 738, 71

Smith, R., \& Gent, P. 2004, Ocean Component of the Community Climate System Model (CCSM2.0 and 3.0), http://www.cesm.ucar.edu/models/ ccsm3.0/pop/doc/manual.pdf

Smith, R. D., \& McWilliams, J. C. 2003, OcMod, 5, 129

Smith, R. S., Dubois, C., \& Marotzke, J. 2006, JCli, 19, 4719

Stevenson, K. B., Lewis, N. L., Bean, J. L., et al. 2016, PASP, 128, 094401

Turbet, M., Bolmont, E., Leconte, J., et al. 2018, A\&A, 612, A86

Turbet, M., Leconte, J., Selsis, F., et al. 2016, A\&A, 596, A112

Vallis, G. K. 2006, Atmospheric and Oceanic Fluid Dynamics (Cambridge: Cambridge Univ. Press), 745

Vallis, G. K. 2012, Climates and the Oceans (Princeton, NJ: Princeton Univ. Press), 245

Vallis, G. K., \& Farneti, R. 2009, TeNov, 135, 1643

Vogt, S. S., Butler, R. P., Rivera, E. J., et al. 2010, ApJ, 723, 954

Wang, Y., Feng, T., \& Hu, Y. 2014, ApJL, 791, L12

Wang, Y., Liu, Y., Feng, T., et al. 2016, ApJL, 823, L20

Watson, A. J., Vallis, G. K., \& Nikurashin, M. 2015, NatGe, 8, 861

Way, M. J., Del Genio, A., Aleinov, I., et al. 2018, ApJS, 239, 24

Way, M. J., Del Genio, A., Kelley, M., Aleinov, I., \& Clune, T. 2015, arXiv: 1511.07283

Way, M. J., Del Genio, A. D., Kiang, N. Y., et al. 2016, GeoRL, 43, 8376

Winton, M. 2003, JCli, 16, 2875

Wolf, E., \& Toon, O. 2015, JGR, 120, 5775

Wolf, E. T. 2017, ApJL, 839, L1

Wolf, E. T., Shields, A. L., Kopparapu, R. K., Haqq-Misra, J., \& Toon, O. B. 2017, ApJ, 837, 107

Yang, J., \& Abbot, D. S. 2014, ApJ, 784, 155

Yang, J., Cowan, N. B., \& Abbot, D. S. 2013, ApJL, 771, L45

Yang, J., Leconte, J., Wolf, E. T., et al. 2018, ApJ, submitted

Yang, J., Liu, Y., Hu, Y., \& Abbot, D. S. 2014, ApJL, 796, L22

Yang, J., Leconte, J., Wolf, E. T., et al. 2016, ApJ, 826, 222

Zalucha, A. M., Michaels, T. I., \& Madhusudhan, N. 2013, Icar, 226, 1743 